\title{
Observational constraints on the composition and nature of comet D/Shoemaker-Levy 9
}

\author{
By J A CQUES CROVISIER
}

Observatoire de Paris-Meudon, CNRS URA 1757, F-92195 Meudon, France

What did the break-up of comet Shoemaker-Levy 9 (SL9) and its subsequent impact on Jupiter teach us about the nature and constitution of this comet? The break-up of the comet apparently triggered activity of the fragments. Although a dust coma was continuously present around the fragments that orbited Jupiter, spectroscopic observations did not reveal any sign of gas. The impact itself was so energetic that most molecules of the impactor were dissociated and that any chemical memory was lost. Ultraviolet and visible spectroscopy of the impact sites revealed emission lines from several atoms, giving potential information on elemental abundances. However, the fact that both neutral and ionized atoms are emitting, and that both fundamental and inter-system lines are present, suggest that the medium is out-of-equilibrium and that emitting mechanisms other than simple resonance fluorescence are at work. Ultraviolet, infrared, and radio spectroscopy revealed lines of several molecular species, in emission and/or absorption, that are not normally present in Jupiter's upper atmosphere. In the visible, dark spots due to aerosols developed at the impact sites. It is not clear at the present time which part of this material is coming from preserved impactor material, from the recombination of the dissociated impactor material, from reactions between the impactor's and Jupiter's material, or from material coming from the lower layers of Jupiter's atmosphere. Realistic modelling of the impacts and of the following chemical reactions will be necessary to address all these issues. In this chapter, we will review the observational clues to the composition and structure of SL9 in the context of our knowledge of the composition and structure of comets and asteroids.

\section{Introduction}

The usual way to study the chemical nature of comets is by remote sensing, through the spectroscopic study of the volatile constituents that sublime under solar heating, and of the small dust particles that are shed during this sublimation process. Such observations, however, are biased towards the study of active regions of the nucleus surface.

We now have a global idea of the composition of cometary volatiles using spectroscopy ranging from radio to UV wavelengths (Crovisier 1994). This knowledge, however, is biased towards comets observed around $1 \mathrm{AU}$, where their activity is governed by water sublimation. Due to sublimation fractionation, the composition of released cometary gases depends upon the heliocentric distance and does not reflect directly the composition of cometary ices.

On special occasions, it was possible to observe sungrazing comets that passed so near the Sun that part of their refractory component was vaporized. In the past century, the spectrum of the Great Comet of 1882 was observed visually by Copeland \& Lohse (1882), revealing the emission lines of several metals. More recently, comet Ikeya-Seki (1965 VIII) was the subject of extensive spectroscopic observations (e.g., Preston 1967) when it passed at $0.0078 \mathrm{AU}$ from the Sun.

The in situ exploration of comet Halley allowed one to investigate the elemental composition of its dust by mass spectroscopy and to evaluate the elemental relative abundances from carbon to nickel (Jessberger \& Kissel 1988); they were found close to the solar 
values. This study was restricted to small-size dust particles. If cometary nuclei contain a population of large refractory particles, their composition is still unknown.

The impact of comet Shoemaker-Levy 9 (D/1993 F2 in the new-style designation of comets; hereafter SL9) on Jupiter gave us the unique occasion to witness the complete disruption of a comet into its elemental constituents and, potentially, to study the bulk composition of its nucleus.

The present chapter reviews the observations of SL9 and of its impact on Jupiter and discusses their implications for the composition and structure of the impactors. To set the stage, Section 2 summarizes our present knowledge of the composition and structure of comets and primitive asteroids. Section 3 is devoted to the pre-impact observations: imaging, photometry, spectroscopy. The study of the disruption of SL9 itself, which gives crucial clues to the structure of this body, is not treated here, since it is the subject of the chapter by Sekanina. Section 4 discusses impact and post-impact observations. The impact itself and its energetics inform us on the mass and density of the impactors; this topic will be only briefly evoked (Section 4.1), since it is the subject of several observational and theoretical reviews in this book. Spectroscopic observations revealed atomic lines (Section 4.2) and molecular signatures (Section 4.3); the observations of these latter are only summarized, since they are presented in detail in the chapter by Lellouch. Section 4.3 draws the consequences of the observations of aerosols on the impact sites, which is also the topic of the chapter by West. In Section 4, we attempt to draw the consequences of the observed molecular and atomic abundances on the composition of SL9 and its nature. Section 5 concludes this review and advocates future work.

\section{The composition of "conventional" comets and asteroids}

The composition of comets is known from the analysis of the output of their activity. For about a century, our investigations were limited to remote sensing by visible spectroscopy. This allowed us to identify several of the dissociation products-radicals, ions, atoms-of the volatile molecules sublimed from the nucleus. We then had to guess the identity of their parent molecule. Only recently was it possible to directly observe these parent species, benefitting from the development of spectroscopy at UV, infrared and radio wavelengths. Our present knowledge of the composition of cometary volatiles is summarized in Fig. 1. More details on the derivation of the composition of cometary ices can be found in Festou et al. (1993), Arpigny (1994) and Crovisier (1994).

An important fraction of cometary material is in the form of refractories, which are partly released as dust particles. An indication of its nature is revealed by the existence of the spectral signature of silicates in the infrared. The space probes to comet Halley had the unique opportunity - up to now-to measure by mass spectroscopy the elemental composition of cometary dust, as reported in Table 1. Another unique event was the observation of the sungrazing comet Ikeya-Seki (1965 VIII), which passed so close to the Sun (0.0078 UA) that part of its refractory component was vaporized and dissociated into atoms. The fluorescence lines of many metals and heavy atoms were then observed in its spectrum ( $\left.\mathrm{Na}, \mathrm{K}, \mathrm{Ca}, \mathrm{Ca}^{+}, \mathrm{V}, \mathrm{Cr}, \mathrm{Mn}, \mathrm{Fe}, \mathrm{Co}, \mathrm{Ni}, \mathrm{Cu}\right)$.

There are many issues still pending concerning the composition of comets. The relative abundances of volatiles are usually observed at heliocentric distances of the order of $1 \mathrm{AU}$, where cometary activity is controlled by water sublimation. They may differ from the relative abundances of cometary ice within the nucleus because of fractionation during the sublimation process and because the most volatile species may be already depleted from sublimation at larger heliocentric distances. Indeed, recent radio observations of $\mathrm{P} /$ Schwassmann-Wachmann 1 (hereafter $\mathrm{P} / \mathrm{SW} 1$ ), an active comet with a nearly circular 


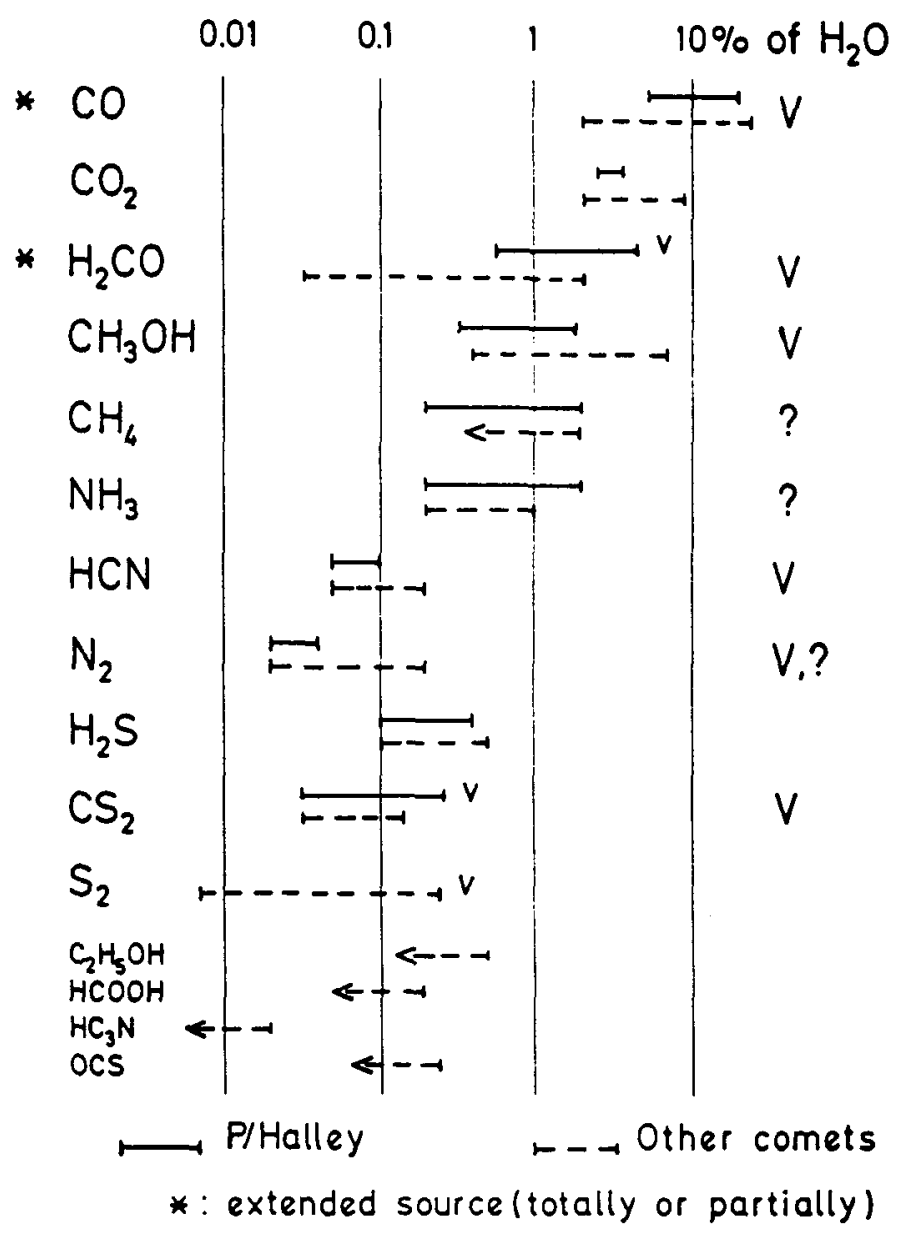

FIGURE 1. Relative abundances of the cometary volatiles expressed in percentage of the water content, by number. " $v$ " indicates temporal variability in a given comet, and "V" in the last column refers to variation from comet to comet. From Arpigny (1994).

orbit at $r_{h} \simeq 6 \mathrm{AU}$ (i.e., beyond Jupiter), revealed that its activity may be governed by $\mathrm{CO}$ sublimation. The cometary activity which is observed far from the Sun-now revealed in more and more comets with the increasing sensitivity of modern techniquesis presumably due to the sublimation of such very volatile species.

Refractories have only been partially sampled in the sungrazing comet Ikeya-Seki and more thoroughly in $\mathrm{P} / \mathrm{Halley}$. In fact, only a very limited fraction of the cometary material (volatiles or refractories) has been sampled either by remote sensing or by in situ analysis. We thus have no direct idea of the bulk composition of comet nuclei. In particular, large size dust particles (rocks)-the existence of which is attested by radar and radio continuum observations-practically escaped all investigations.

To further complicate the situation, there may be a high degree of diversity of composition among comets resulting from their formation in different regions of the solar nebula. The direct measurements of parent volatiles are still sparse (although they in- 


\begin{tabular}{|c|c|c|c|c|c|}
\hline \multirow[t]{2}{*}{ Element } & \multirow[t]{2}{*}{ Ikeya-Seki $†$} & \multicolumn{2}{|r|}{ P/Halley } & \multirow[t]{2}{*}{ Solar system } & \multirow[t]{2}{*}{ CI-chondrite } \\
\hline & & dust & dust + ice & & \\
\hline $\mathrm{H}$ & & 2025. & 4062. & $2.6 \times 10^{6}$ & 492. \\
\hline $\mathrm{Li}$ & $\dagger$ & & & 0.0053 & 0.0053 \\
\hline $\mathrm{C}$ & & 814. & 1010. & 940. & 70.5 \\
\hline$N$ & & 42. & 95. & 291. & 5.6 \\
\hline 0 & & 890. & 2040 & 2216. & 712. \\
\hline $\mathrm{Na}$ & $t$ & 10. & 10. & 5.3 & 5.3 \\
\hline $\mathrm{Mg}$ & $(=100.0)$ & $=100.0$ & $=100.0$ & $=100.0$ & $=100.0$ \\
\hline $\mathrm{Al}$ & & 6.8 & 6.8 & 7.9 & 7.9 \\
\hline $\mathrm{Si}$ & & 185. & 185. & 93. & 93. \\
\hline$P$ & & & & 1.0 & 1.0 \\
\hline S & & 72. & 72. & 48. & 48. \\
\hline K & $\dagger$ & 0.2 & 0.2 & 0.35 & 0.35 \\
\hline $\mathrm{Ca}$ & & 6.3 & 6.3 & 5.7 & 5.7 \\
\hline $\mathrm{Ti}$ & $<0.02$ & 0.4 & 0.4 & 0.22 & 0.22 \\
\hline V & 0.01 & & & 0.027 & 0.027 \\
\hline $\mathrm{Cr}$ & 0.08 & 0.9 & 0.9 & 1.3 & 1.3 \\
\hline $\mathrm{Mn}$ & 0.5 & 0.5 & 0.5 & 0.89 & 0.89 \\
\hline $\mathrm{Fe}$ & 84. & 52. & 52. & 84. & 84. \\
\hline $\mathrm{Co}$ & 0.4 & 0.3 & 0.3 & 0.21 & 0.21 \\
\hline $\mathrm{Ni}$ & 7.2 & 4.1 & 4.1 & 4.6 & 4.6 \\
\hline $\mathrm{Cu}$ & 0.2 & & & 0.049 & 0.049 \\
\hline
\end{tabular}

$\dagger$ In addition, Preston (1967) determined abundances relative to $\mathrm{Na}$ of $1.6 \times 10^{-3}$ for $\mathrm{K}$ and $<2.5 \times 10^{-5}$ for $\mathrm{Li}$.

TABLE 1. Average elemental abundances measured in comet Ikeya-Seki (from Arpigny et al. 1995), in Halley's dust grains and in the whole comet dust and volatiles (from Jessberger \& Kissel 1991), and in other solar system objects (from Anders \& Grevesse 1989). The abundances are normalized to $\mathrm{Mg}$ (to the solar system abundance of Fe for Ikeya-Seki, in which $\mathrm{Mg}$ was not observed).

dicate strong variations of the $\left[\mathrm{CH}_{3} \mathrm{OH}\right] /\left[\mathrm{H}_{2} \mathrm{O}\right]$ ratio, for instance). The investigation of the radical abundances in significant samples reveals relative abundance variations of $\mathrm{OH}, \mathrm{CN}, \mathrm{C}_{2}, \mathrm{C}_{3}$ and $\mathrm{NH}$. Thus A'Hearn et al. (1996) could separate two classes of comets from the observations of their radicals: "typical" and " $\mathrm{C}_{2}$-depleted"; these latter being related to Jupiter-family comets. Also suspected is the heterogeneity of cometary nuclei, resulting from the possible aggregation of planetesimals of different origins.

The density of cometary nuclei is not well known. The nucleus size is known directly from imaging only for $\mathrm{P} / \mathrm{Halley}$. It can, in principle, be determined from photometry, but practically it is very difficult to disentangle the respective contributions of the nucleus and of the dust coma for an active comet. On the other hand, the only way to have access to the nucleus mass at the present time is by relating the evaluation of nongravitational forces-due to anisotropic outgassing-to their perturbating effect on the cometary orbits. This method is still highly model-dependent. Available evaluations lead to densities in the range $0.2<\rho<0.9 \mathrm{~g} \mathrm{~cm}^{-3}$ for $\mathrm{P} /$ Halley and very few other comets (Festou et al. 1993, Rickman 1994).

Since there is no release of material from bona fide asteroids, information on their composition can only come from spectroscopy of the solid minerals at their surface. This is of little help, since this material is probably weathered and has little to do with the 
internal composition of these differentiated bodies. However, it is usually believed that most meteorites recovered on Earth are of asteroidal origin, thus providing models for asteroidal matter. But the precise links between meteorites and asteroids are not easy to establish.

Although asteroid sizes are known for a large sample of objects, there is little information on their masses which could lead to density determinations. Evaluations from mutual perturbations are rare and inaccurate (Hoffmann 1989). Recently, the Galileo probe, when imaging (243) Ida, discovered a second component, Dactyl. Unfortunately, the orbit of the binary system could not be accurately determined and the density of Ida is poorly constrained: $2.1<\rho<3.1 \mathrm{~g} \mathrm{~cm}^{-3}$ (Belton et al. 1995). This indicates a chondritic composition and/or a highly porous structure.

Table 1 shows that there are little differences between different objects with regard to the abundances of heavy elements; all abundances are close to "cosmic". This is no longer the case for light elements; these elements are likely to be deposited in highly volatile components; their abundances are thus highly dependent on the physical conditions of the formation and evolution of these various objects.

\section{Pre-impact observations}

\subsection{Imaging and photometry}

The pre-impact images of SL9 were obtained at several observatories: e.g., Chernova et al. (1995) with the ESO 1-m; Cochran et al. (1995) at McDonald; Colas et al. (1995) at Pic-du-Midi; Jewitt (1995a) at the Mauna Kea telescopes; Scotti et al. (1994) at the Spacewatch station; Stüwe et al. (1995) with the ESO-NTT; Trilling et al. (1995) at Calar-Alto; Weaver et al. $(1994,1995)$ with the HST; R. M. West et al. (1995) with several ESO telescopes.

Tancredi \& Lindgren (1994) have reported a negative search for comets in the vicinity of Jupiter undertaken at ESO in March-April 1992, i.e., one year before the discovery of SL9 and several months before its disruption by Jupiter. From their limiting magnitude $B=21.5$, they infer an upper limit of $7.2 \mathrm{~km}$ on the radius of SL9 nucleus before its disruption.

\subsubsection{Sizes of nuclei from the images}

Retrieving the nucleus sizes from the images is a difficult problem. The nuclei are not resolved. One could hope to separate the relative contributions of the star-like nucleus and of the dust distribution from high-resolution images. Assuming an albedo similar to that observed for other comet nuclei, it would then be possible to derive the nucleus cross-section. The problem is that the dust distribution does not follow the typical $1 / r$ law observed in other comets; it is steeper and curved, precluding an easy extrapolation to the unresolved nuclear region. To further complicate the problem, we may have to deal with multiple nuclei (or even with swarms of nuclei, as postulated by Xie et al. 1995).

From ground-based observations, Jewitt (1995a) gave an upper limit of $1 \mathrm{~km}$ to the fragment sizes. From the HST images, Weaver et al. $(1994,1995)$ derived sizes ranging from 0.6 to $4.1 \mathrm{~km}$ in diameter for the various fragments (assuming an albedo of 0.04 ), which are in fact conservative upper limits to the true values. Sekanina (1995b) independently tried to deconvolve the HST images and argued that the fragments could have, in fact, multiple secondary nuclei in addition to a large main component. 


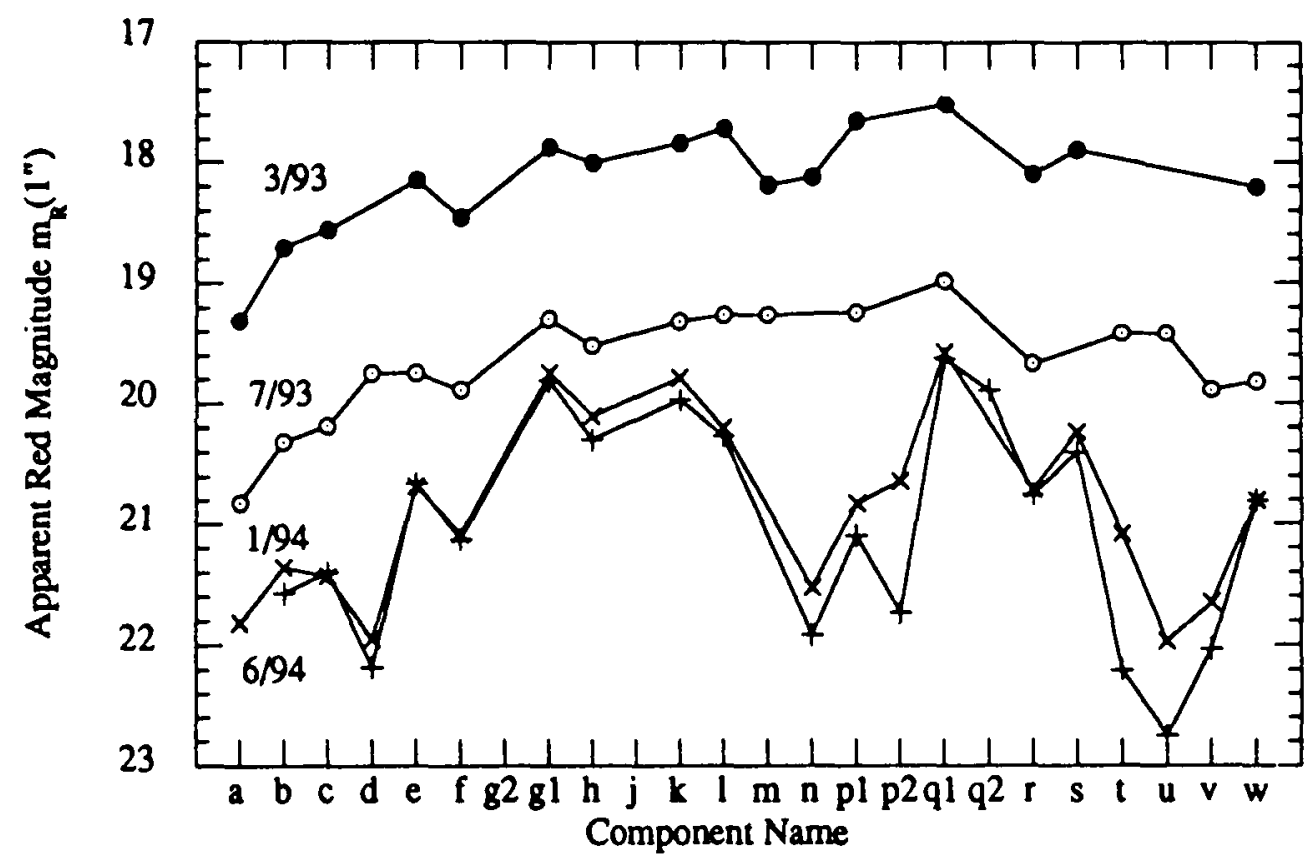

FIGURE 2. The evolution of the fragment brightness: R-filter photometry of the SL9 components with a $1^{\prime \prime}$ radius aperture, at three different epochs, from Jewitt (1995a). All components fade as dust dissipates; however, different components fade at different rates.

\subsubsection{Activity}

It is of paramount importance to determine whether the comae observed around every fragment are the remnants of the dust outburst which followed the 1992 break-up, or are due to continuous activity. This is still a controversial point which has been discussed by several authors (Sekanina et al. 1994; Weaver et al. 1994, 1995; Jewitt 1995a; Sekanina $1995 \mathrm{a} ; \mathrm{R}$. A. West, this volume).

The evolution of the fragment brightnesses, as observed by Jewitt (1995a), is shown in Fig. 2. There is undoubtedly an important fading of all components, revealing a decrease of activity, or even the dissipation of dust in the absence of any activity. It has been noted that in the absence of activity, the persistence of the central coma can only be explained by the presence of large-size (centimetre-size) particles. From ground-based observations, Jewitt (1995a) estimated that the observed dust distribution could be consistent with a dust production rate of no more than $1 \mathrm{~kg} \mathrm{~s}^{-1}$ for the largest components, whereas the analysis of the HST observations by Weaver et al. (1995) yielded a production rate of at most $5 \mathrm{~kg} \mathrm{~s}^{-1}$ (the difference between these two evaluations could be ascribed to differences in the dust models rather than to the observations themselves).

Each component being accompanied by its own tail, the tail morphology and evolution are important clues to the dust properties and production. Most of the time, the tail's orientation was close to the opposite Sun direction, as are all well-behaved cometary tails due to a continuous dust production under the influence of solar radiation pressure. Just a few days before the impacts, the tails and dust distributions were deformed and elongated along the comet-Jupiter direction (R. M. West et al. 1995), presumably under the influence of Jovian tidal forces. At the moment of opposition at the end of April 


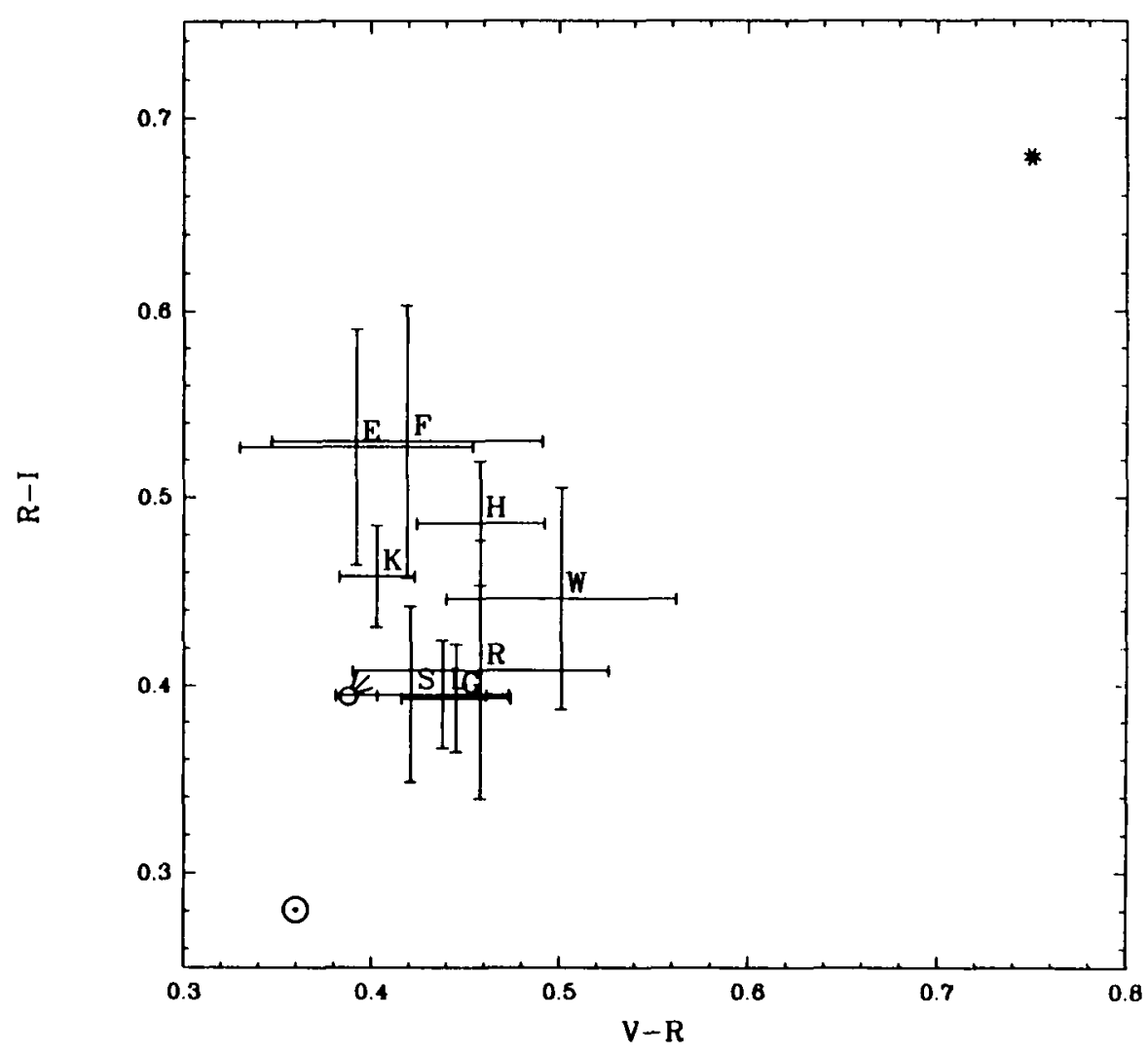

FIgURE 3. The colours of the fragments of SL9, plotted from the Mauna Kea data of mid-January 1994 reported by Meech and Weaver (1995); only the fragments with the best data are plotted, with $\pm 1 \sigma$ error bars; symbols indicate the colours of the Sun $(\odot)$, of a typical comet $(\odot<)$ and of Pholus $\left(^{*}\right)$.

1994 , the phase angle was very close to 0 . One would then expect a rapid rotation of the tail's position angle, following the anti-Sun direction. It was noted by several observers (Jewitt 1995a, Scotti et al. 1994) that this was not exactly the case. Detailed modelling of this process, in order to retrieve dust properties and the evolution of dust production from available images, is highly desirable (see also Sekanina, this volume).

\subsubsection{Were all fragments alike?}

In addition to their difference in sizes, in activity (production of dust, fading, further splitting), the SL9 fragments might have differences in composition. Such an effect might result from the formation of the initial SL9 nucleus itself, like from the accretion of planetesimals of different origins. A test of this could come from broadband photometric or spectrophotometric observations, which could reveal differences in the colours of the fragments. Such observations were performed by several teams: Chernova et al. (1995); Cochran et al. (1994), Rauer and Osterloh (1995); Meech and Weaver (1995); Trilling et al. (1995). No significant differences between the fragments were reported.

For example, Fig. 3 shows the colours of the fragments as observed by Meech and Weaver (1995) at Mauna Kea. The results are dispersed and some fragments have colours 


$\begin{array}{llrr}\text { Instrument } & \text { Date } & \begin{array}{r}Q[\mathrm{OH}] \\ {\left[\mathrm{s}^{-1}\right]}\end{array} & \begin{array}{r}Q[\mathrm{CN}] \\ {\left[\mathrm{s}^{-1}\right]}\end{array} \\ \text { HST 2.2-m } & \text { Jul. 93-Jul. 94 } & <1-2 \times 10^{27} & \\ \text { McDonald 2.7-m } \ddagger & \text { Feb. + Mar. 94 } & <8.4 \times 10^{26} & <1.7 \times 10^{24} \\ \text { CFHT 3.6-m } & \text { 5-6 Mar. 94 } & & <3 \times 10^{24} \\ \text { Keck 10-m } & \text { 10 Apr. 94 } & <3 \times 10^{23} \\ \text { ESO/NTT 3.5-m } & \text { 1-2 Jul. 94 } & <1.8-2.8 \times 10^{23}\end{array}$

$\dagger$ References. HST: Weaver et al. 1994, 1995; McDonald: Cochran et al. 1994; ESO/NTT: Stüwe et al. 1995; CFHT and Keck: Jewitt, Luu and Owen, private communication.

$\ddagger$ These limits pertain to a combination of several fragments.

TABLE 2. Spectroscopic limits $(3 \sigma)$ on gas production rates in SL9 prior to the impacts.

differing by more than the $\pm 1 \sigma$ errors, but not much more. This does not seem to be a convincing clue for diversity among the fragments. If real, these effects would rather reflect different size distributions of the dust particles. The figure shows, however, that the dust is definitely redder than the Sun and marginally redder than the average comet. It is not, however, as red as the enigmatic distant asteroid Pholus.

\subsection{Spectroscopy}

Several sensitive pre-impact spectroscopic searches for signs of gas in the visible (CN and $\mathrm{CO}^{+}$) and in the near-UV (OH) were undertaken from the ground and with the HST. They are summarized in Table 2. They were negative. Upper limits on the production rates were $Q[\mathrm{OH}]<1-2 \times 10^{27} \mathrm{~s}^{-1}$ and $Q[\mathrm{CN}]<2-3 \times 10^{23} \mathrm{~s}^{-1}$. The upper limit on CN would correspond to $Q[\mathrm{OH}]<10^{26} \mathrm{~s}^{-1}$ if one assumes $Q[\mathrm{OH}] / Q[\mathrm{CN}] \simeq 350$, as for comets observed at $r_{h} \simeq 1 \mathrm{AU}$ (A'Hearn et al. 1995), which may be invalid at $5 \mathrm{AU}$.

Searches for CO at radio wavelengths were also negative at the CSO (Jewitt \& Senay 1994, private communication), at the SEST (Rickman 1994, private communication), as well as at IRAM where observations were conducted at the very moment preceding the impacts (Festou \& Lellouch 1994, private communication). No limits on the corresponding $\mathrm{CO}$ production rate were given up to now; they are presumably on the order of several $10^{28} \mathrm{~s}^{-1}$ ).

A comparison can be made with the activity of other distant comets (see Huebner et al. [1993] for a review): spectroscopic detections are very rare for comets at $r_{h}>5 \mathrm{AU}$. CN was detected in Chiron at $10 \mathrm{AU}, \mathrm{P} / \mathrm{SW} 1$ at $6 \mathrm{AU}$ and $\mathrm{P} / \mathrm{Halley}$ when it was at $4.8 \mathrm{AU}$ pre-perihelion; $\mathrm{CO}^{+}$was only found in $\mathrm{P} / \mathrm{SW} 1$. Recently, the radio rotational lines of CO were observed in P/SW1 (Senay \& Jewitt 1994; Crovisier et al. 1995) and in the long-period comet C/1995 Q1 (Hale-Bopp) (Matthews et al. 1995; Rauer et al. 1995); the corresponding $\mathrm{CO}$ production rate in these comets is as large as $2-5 \times 10^{28} \mathrm{~s}^{-1}$.

At 5.4 AU the equilibrium temperature of a rapidly rotating nucleus is $120 \mathrm{~K}$, which is not sufficient to allow the sublimation of water: the sublimation rate, which is of the order of $10^{17}$ water molecules $\mathrm{cm}^{-2} \mathrm{~s}^{-1}$ at $1 \mathrm{AU}$, drops to $3 \times 10^{9} \mathrm{~cm}^{-2} \mathrm{~s}^{-1}$-more than seven orders of magnitudes lower - at $5.4 \mathrm{AU}$. Other effects which have been invoked to explain distant activity are: (i) a slowly rotating nucleus or (ii) small icy grains which could still be heated up to $150 \mathrm{~K}$ at these distances and have significant water ice sublimation rates $\left(\sim 10^{14} \mathrm{~cm}^{-2} \mathrm{~s}^{-1}\right) ;(i i i)$ an internal source of energy such as the exothermic conversion of amorphous ice into crystalline ice; or (iv) the sublimation of 
more volatiles species, which scales at $r_{h}^{-2}$ up to much larger values of $r_{h}$. The recent detection of a large $\mathrm{CO}$ production rate in distant comets shows that the last process is indeed at work.

As stated in Section 3.1.2, it is not yet clear from the analyses of the SL9 images whether the release of dust was continuous or restricted to the period following the July 1992 disruption. If continuous, the dust production rate was very low (no more than $1-5 \mathrm{~kg} \mathrm{~s}^{-1}$ per fragment). When scaled to $\mathrm{P} / \mathrm{SW} 1$ according to its dust production, the expected production rate of CO from the fragments of SL9 is too low to permit detection $\left(\mathrm{P} / \mathrm{SW} 1\right.$ has a dust production rate of the order of $10-100 \mathrm{~kg} \mathrm{~s}^{-1}$ and $Q[\mathrm{CO}] \simeq$ $5 \times 10^{28} \mathrm{~s}^{-1}$ ). At $4.8 \mathrm{AU}$ from the Sun, $Q[\mathrm{CN}]$ was $3 \times 10^{24} \mathrm{~s}^{-1}$ for P/Halley (Wyckoff et al. 1985 ) whereas its dust production was $\sim 3 \mathrm{~kg} \mathrm{~s}^{-1}$ as estimated by Weaver et al. (1995), but this was at $r_{h}$ somewhat smaller than SL9. Thus, the non-detection of any gas signature in the spectrum of SL9 is not really surprising.

Another peculiar example of distant activity is that developed by the dynamically new comet Bowell (1982 I). This comet showed a significant production of $\mathrm{OH}(7.5 \times$ $10^{28} \mathrm{~s}^{-1}$ ) pre-perihelion as far as $5.25 \mathrm{AU}$ from the Sun, which did not increase much when the comet reached perihelion at $3.26 \mathrm{AU}$ (A'Hearn et, al. 1984). This unusually high water production rate at this heliocentric distance was interpreted as coming from the sublimation of water from icy grains; the very large surface of the grains compensates for the very low sublimation rate at this distance, ensuring an efficient water production. At similar heliocentric distances, the dust production parameter $A f \rho \dagger$ was $\simeq 7000 \mathrm{~cm}$ for comet Bowell (A'Hearn et al. 1984) and $\simeq 200-500 \mathrm{~cm}$ for the strongest fragments of SL9 in early 1994, from photometric measurements of Cochran et al. (1994) and Jewitt (1995a and Fig. 2). If $Q[\mathrm{OH}] / A f \rho$ were the same for both objects, one would expect $Q[\mathrm{OH}] \simeq 5 \times 10^{27} \mathrm{~s}^{-1}$ for the strongest fragments of SL9, which is significantly more than the observed upper limits (table 2). It can be inferred that the grains of SL9 had a different nature from those of comet Bowell, or that they lost their icy component in the first months following the disruption of the comet by Jupiter in July 1992.

A puzzling flash of $\mathrm{Mg}^{+}$emission (doublet near $280 \mathrm{~nm}$ ) was fortuitously observed with the HST/FOS on fragment G on 14 July 1994, four days before impact (Weaver et al. 1995; Feldman et al. 1996). This emission, which decayed with a time constant of one minute, was followed by an increase of the dust scattered emission. This transient event may be associated with the entry of the fragment in Jupiter's magnetosphere, a possible source mechanism being ion sputtering of the dust surrounding the fragment, as suggested by Feldman et al. (1995).

\section{Impact and post-impact observations}

Cometary molecules impacting at a velocity of $60 \mathrm{~km} \mathrm{~s}^{-1}$ have a kinetic energy about two orders of magnitude higher than their chemical binding energy. Therefore, not only will they be dissociated, but the excess energy of their fragments will be high enough to initiate several chemical reactions (dissociations) within Jupiter's atmosphere. In the shock (or hot) chemistry which will follow, all memory of the initial chemical composition of the impactor will be lost (e.g., Borunov et al. 1995).

However, we cannot exclude the possibility that part of the initial cometary molecules may be preserved (for instance, after successive fragmentations, small particles might

$\dagger$ The parameter $A f \rho$, first introduced by A'Hearn et al. (1984), is the product of dust albedo $A$, dust filling factor $f$ and field of view diameter $\rho$. It is homogeneous to a distance. It can be straightforwardly derived from photometry and it is a convenient measure of the dust production rate. 
1

$2 a$

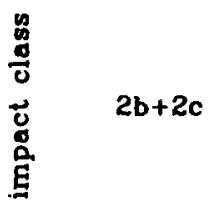

3
A

DN

$\mathbf{B}$
L $\mathbf{K G}_{\text {, }}$

C E H

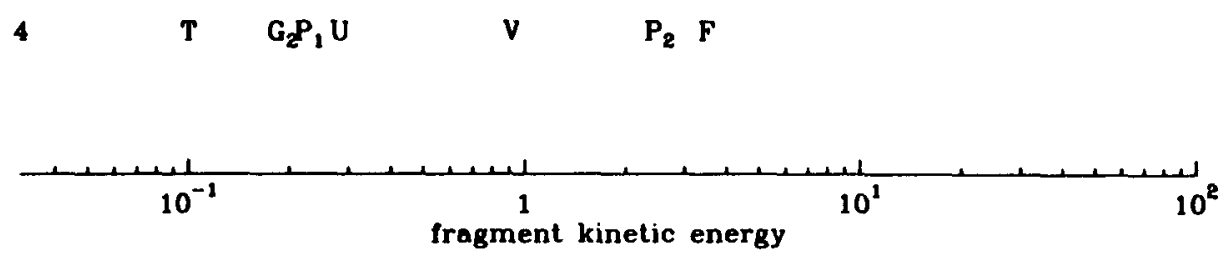

Figure 4. The classes of the impacts, as defined by Hammel et al. (1995), as a function of the fragment relative kinetic energies, evaluated by Weaver et al. (1995) from the brightnesses measured by the HST.

be decelerated without being destroyed). The proportion of such preserved material is unknown, but it is presumably small compared to the amount of chemically processed material.

Thus, clues to the chemical composition of the impactor must be searched for in the elemental abundances. The upper atmosphere of Jupiter is devoid of heavy elements, of oxygen and sulphur compounds, and is depleted in nitrogen. The detection of such elements after the impacts could betray the presence of SL9 material. However, such clues might be masked by material coming from the deep atmosphere of Jupiter (and especially from the $\mathrm{NH}_{3}, \mathrm{NH}_{4} \mathrm{SH}$ and water clouds) if the fragments went so far.

\subsection{Clues from the energetics of the impacts}

The manifestations of the impact phenomena, such as their light curves, the fireball brightnesses and sizes, and the depths to which the impactors penetrated into Jupiter's atmosphere, critically depend upon the sizes and density of the fragments. This is discussed in the theoretical chapters on the physics of the impacts (chapters by Crawford, Mac Low, Zahnle, this volume). For instance, Zahnle \& Mac Low (1995) estimated from the light curve of its impact that fragment $R$ had diameter $450-500 \mathrm{~m}$ and mass $\sim 2-3 \times 10^{13} \mathrm{~g}$.

It was noted by Weaver et al. (1995) and by several other observers that the brightest fragments did not yield the strongest impacts. This is illustrated in Fig. 4 which shows the classes of the impacts, as defined by Hammel et al. (1995) on the basis of the aspects of the impact sites, as a function of the pre-impact brightnesses of the fragments as measured by the HST. Although the figure shows a clear correlation between these two 


\begin{tabular}{|c|c|c|c|c|}
\hline species & line or band & $\begin{array}{r}\text { frequency } \\
\text { wavelength }\end{array}$ & instrument ${ }^{\dagger}$ & impact \\
\hline radio: & & {$[\mathrm{GHz}]$} & & \\
\hline $\mathrm{H}_{2} \mathrm{O}$ & $6_{16}-5_{23}$ & 22 & Medicina & $\mathrm{E}$ \\
\hline $\mathrm{HCN}$ & $1-0$ & 89 & IRAM-inter & A \\
\hline $\mathrm{CO}$ & $1-0$ & 115 & IRAM-30m & several \\
\hline OCS & $18-17$ & 219 & IRAM-30m & K W \\
\hline $\mathrm{CO}$ & $2-1$ & 230 & IRAM-30m, SEST & several \\
\hline $\mathrm{CS}$ & $5-4$ & 245 & IRAM-30m & several \\
\hline $\mathrm{HCN}$ & $3-2$ & 266 & JCMT & several \\
\hline $\begin{array}{l}\mathrm{HCN} \\
\text { infrared: }\end{array}$ & $4-3$ & $\begin{array}{r}345 \\
{[\mu \mathrm{m}]}\end{array}$ & JCMT & several \\
\hline $\mathrm{H}_{2} \mathrm{O}$ & rot. & $23.9,22.6$ & KAO/KEGS & G K \\
\hline $\mathrm{HCN}$ & $\nu_{2}$ & 13.4 & NASA/IRTF & several \\
\hline $\mathrm{C}_{2} \mathrm{H}_{4}$ & $\nu_{7}$ & 11.0 & NASA/IRTF & $\mathrm{K}$ \\
\hline silicates & & $\simeq 10.0$ & Palomar & $\mathbf{R}$ \\
\hline $\mathrm{PH}_{3}$ & $\nu_{2}$ & 10.1 & Palomar & $\mathrm{L}$ \\
\hline $\mathrm{H}_{2} \mathrm{O}$ & $\nu_{2}$ & 6.6 & KAO/HIFOGS & R W \\
\hline $\mathrm{H}_{2} \mathrm{O}$ & $\nu_{2}$ & 7.7 & KAO/KEGS & G K \\
\hline $\mathrm{CO}$ & $\Delta v=1$ & 4.7 & several & several \\
\hline $\mathrm{H}_{2} \mathrm{O}$ & $\nu_{3}$ & 2.7 & Galileo/NIMS & G R \\
\hline $\mathrm{CO}$ & $\Delta v=2$ & 2.3 & several & several \\
\hline $\mathrm{H}_{2} \mathrm{O}$ & $\nu_{3}, \nu_{2}+\nu_{3}$ & $2.38,2.0$ & AAT & $\mathrm{C} \mathrm{K}$ \\
\hline $\mathrm{NH}_{3}$ & several & & several & several \\
\hline $\mathrm{CH}_{4}$ & several & & several & several \\
\hline $\mathrm{H}_{2}$ & several & & several & several \\
\hline $\mathrm{H}_{3}^{+}$ & several & & several & several \\
\hline
\end{tabular}

$\dagger$ References to the Table. Medicina: Montebugnoli et al. 1995; IRAM-inter: Wink et al. 1994; IRAM-30m: Lellouch et al. 1995; SEST: Bockelée-Morvan et al. 1995; JCMT: Marten et al. 1995; KAO/KEGS: Bjoraker et al. 1995; KAO/HIFOGS: Sprague et al. 1995; NASA/IRTF: Bézard et al. 1995, Griffith et al. 1995, Orton et al. 1995; AAT: Crisp \& Meadows 1995; Galileo/NIMS: Carlson et al. 1995; Palomar: Conrath et al. 1995; Nicholson et al. 1995.

TABLE 3. Detected radio and infrared spectral lines and bands during the SL9/Jupiter impact event.

parameters, some fragments depart from this law: $\mathrm{A}$ and $\mathrm{C}$ gave relatively stronger impacts, whereas the impacts of Q1 and Q2 were relatively fainter. This could betray a diversity among the fragments; if the impact class corresponds to the fragment mass, and the fragment brightness to the fragment size, this would suggest a diversity of densities. But it could also correspond to a pre-fragmentation of some of the fragments, or to diversity in their emission of dust.

\subsection{Clues from atomic lines}

Emissions of atomic lines were reported in the UV from the HST (Noll et al. 1995) and the IUE (Prangé et. al. 1994), and in the visible from several ground-based observations (Catalano et al. 1995; Costa et. al. 1994; Fitzsimmons et al. 1995; Roos-Serote et al. 1995a, b). They are listed in Table 4. It should be noted that several unidentified lines were also observed that might be due to atoms or ions.

The atomic lines observed in the visible from the ground (Figs. 5, 6 and 7) were emitted in the splashback phase of the collision, as could be determined from the time-resolved 
species line or band

$\begin{array}{ll}\text { visible: } & \\ \mathrm{Fe} & a^{5} F-z^{7} D^{0} \\ \mathrm{~K} & 4 s^{2} S-4 p^{2} P^{0} \\ \mathrm{Li} & 2 s^{2} S-2 p^{2} P^{0} \\ \mathrm{Ca} & 4 s^{2}{ }^{1} S-4 p^{3} P^{0} \\ \mathrm{H} & \mathrm{H} \alpha \\ \mathrm{Fe} & a^{5} F-z^{7} F^{0} \\ \mathrm{Na} & 3 s^{2} S-3 p^{2} P^{0} \\ \mathrm{Mn} & a^{6} S-z^{8} P^{0} \\ \mathrm{Fe} & a^{5} D-z^{7} D^{0} \\ \mathrm{Mg} & 3 s^{2}{ }^{1} S-3 p^{3} P^{0} \\ \mathrm{Fe} & a^{5} D-z^{7} F^{0} \\ \mathrm{Cr} & a^{7} S-z^{7} P^{0}\end{array}$

ultraviolet:

\begin{tabular}{|c|c|}
\hline $\mathrm{Na}$ & $3 s^{2} S-4 p^{2} P^{0}$ \\
\hline $\begin{array}{l}\mathrm{S}_{2} \\
?\end{array}$ & $B^{3} \Sigma_{u}^{-}-X^{3} \Sigma_{g}^{-}$ \\
\hline $\mathrm{Mg}$ & $3 s^{2}{ }^{1} S-3 p{ }^{1} P^{0}$ \\
\hline $\mathrm{Mg}^{+}$ & $3 s^{2} S-3 p^{2} P^{0}$ \\
\hline $\mathrm{Fe}^{+}$ & $4 s^{6} D-z^{6} D^{0}$ \\
\hline CS & $A^{1} \Pi-X^{1} \Sigma$ \\
\hline $\mathrm{Si}$ & $3 p_{2}{ }^{3} P-4 s^{3} P^{0}$ \\
\hline $\mathrm{Fe}$ & $4 s^{25} D-x^{5} F^{0}$ \\
\hline $\mathrm{NH}_{3}$ & $\tilde{A}^{1} A^{\prime \prime}{ }_{2}-\tilde{X}^{1} A_{1}$ \\
\hline $\mathrm{CS}_{2}$ & $\bar{A}^{1} B_{2}-\bar{X}^{1} \Sigma_{g}^{+}$ \\
\hline $\mathrm{H}_{2} \mathrm{~S}$ & $\bar{A}-\tilde{X}^{1} A_{1}$ \\
\hline $\mathrm{S}$ & $3 p^{4}{ }^{4} S^{0}-4 s^{3} S^{0}$ \\
\hline $\begin{array}{l}\mathrm{Si}^{+} \\
?\end{array}$ & $3 p^{2} P^{0}-3 p^{2}{ }^{2} D$ \\
\hline $\mathrm{Al}^{+}$ & $3 s^{21} S-3 p{ }^{1} P^{0}$ \\
\hline $\mathrm{C}$ & $2 p^{2}{ }^{3} P-3 s{ }^{3} P^{0}$ \\
\hline $\mathrm{H}_{2}$ & $B^{1} \Sigma_{u}^{+}-X^{1} \Sigma_{g}^{+}$(Lyman) \\
\hline $\mathrm{S}^{+}$ & $3 p^{3}{ }^{4} S^{0}-3 p^{4^{4}}{ }^{4} S$ \\
\hline $\mathrm{H}_{2}$ & $C^{1} \Pi_{u}-X^{1} \Sigma_{g}^{+}$(Werner) \\
\hline $\mathrm{H}$ & Ly $\alpha$ \\
\hline $\mathrm{He}$ & $1 s^{1} S-2 p{ }^{1} P^{0}$ \\
\hline
\end{tabular}

$$
\underset{[\mathrm{nm}]}{\text { wavelength }^{\text {instrument }}{ }^{\dagger} \quad \text { impact }}
$$

$\begin{array}{lll}804.8 & \text { OMP } & \text { L } \\ 766.5 & \text { OMP } & \text { L Q } \\ 670.8 & \text { OMP, Brazil } & \text { L } \\ 657.3 & \text { OMP, Brazil, INT } & \text { L } \\ 656.3 & \text { OMP } & \text { L } \\ 635.9 & \text { OMP, Brazil } & \text { L }\end{array}$

589.6 \& 589.0 OMP, Brazil, INT, $\quad \mathrm{H} \mathrm{L} \mathrm{Q}_{1} \mathrm{Q}_{2}$

\section{Catania}

$\begin{array}{lll}543.2 & \text { INT } & \text { L } \\ 511.0 & \text { INT } & \text { L } \\ 457.2 & \text { INT } & \text { L } \\ 437.7 & \text { INT } & \text { L } \\ 425.4 & \text { INT } & \text { L }\end{array}$

$\begin{array}{rll}330.2 & \text { IUE } & \text { A } \\ 309.6 & \text { HST unidentified } & \text { S } \\ 293-255 & \text { HST } & \text { G S } \\ 288.4 & \text { HST unidentified } & \text { S } \\ 285.3 & \text { HST } & \text { S }\end{array}$

$279.6 \& 280.5$ HST $\quad$ pre-impact, $\mathrm{S}$

262. HST S

257.8 HST

$251.5 \& 252.5$ HST $\quad \mathrm{S}$

248.4 HST $S$

230-180 HST G

206.4-187.3 HST S

$\sim 200 \quad$ HST tentative $\quad$ G

180.8-182.0 IUE (or $\mathrm{Si}^{+}$?) $\quad \mathrm{A}$

180.7-181.7 IUE (or $\mathrm{S}$ ?) A

$176 \& 178$ IUE unidentified A

167.1 IUE tentative K

165.7 IUE tentative $\mathrm{K}$

155.-162. IUE K S

125. HST G

123.-130. IUE K S

121.5 IUE K S

58.4 EUVE $\quad \mathrm{Q}_{1}$

$\dagger$ References to the Table. OMP: Roos-Serote et al. 1995a; Brazil: Costa et al. 1994; INT: Fitzsimmons et al. 1995; Catania: Catalano et al. 1995; IUE: Prangé et al. 1994, Ballester et al. 1995; HST: Noll et al. 1995, Weaver et al. 1995, Yelle \& McGrath 1995; EUVE: Gladstone et al. 1995.

TABLE 4. Detected visible and ultraviolet spectral lines and bands during the SL9/Jupiter impact event. 


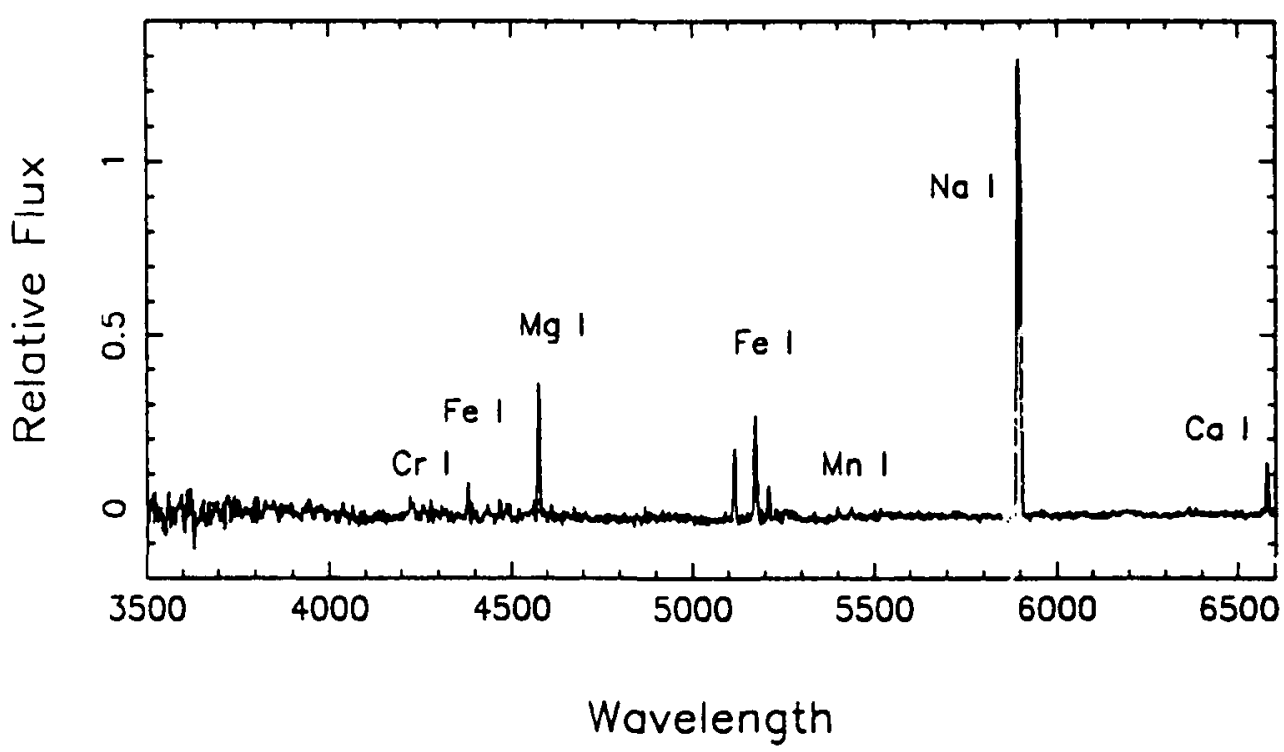

Figure 5. Atomic line emissions observed in the visible with the 2.5-m Isaac Newton Telescope at La Palma observatory in the L-plume on July 19 at 22:35 UT, just after the impact. The wavelength is given in ångstrøms. From Fitzsimmons et al. (1995).

observations of impact L at La Palma (Fitzsimmons et al. 1995). No emission could be detected more than one hour after the impact. The strongest lines of $\mathrm{Na}, \mathrm{Ca}, \mathrm{Fe}$ and even $\mathrm{Li}$ were visible in the raw spectra, whereas weaker lines of $\mathrm{Fe}$ and Ca multiplets and the $\mathrm{H} \alpha$ emission could only be spotted in spectra corrected for the solar reflected spectrum (Roos-Serote et al. 1995b). The IUE observations (Ballester et al. 1995) revealed emission lines and bands of $\mathrm{H}$ Lyman $\alpha$ and $\mathrm{H}_{2}$ in spectra of impact sites $\mathrm{K}$ and $\mathrm{S}$ observed for $\simeq 20$ min around impact times. The far- $\mathrm{UV}$ emission lines of carbon and $\mathrm{Al}^{+}$were tentatively identified in impact $\mathrm{K}$. With the exception of the $\mathrm{Mg}^{+}$flash pre-impact observation (see Section 3.2), the metallic emission lines of $\mathrm{Mg}, \mathrm{Mg}^{+}, \mathrm{Fe}, \mathrm{Fe}^{+}$and $\mathrm{Si}$ detected in the UV by the HST (Fig. 8) were observed in a field encompassing the sites of three-day old impact $\mathrm{G}$ and of the fresh impact $\mathrm{S}$ just $45 \mathrm{~min}$ after its formation. It is thus likely that these lines are coming from new impact $S$ rather than old impact $G$.

All these observations remind us of the observed spectra of sungrazing comets which showed the lines of several metals $(\mathrm{Na}, \mathrm{K}, \mathrm{Ca}, \mathrm{V}, \mathrm{Cr}, \mathrm{Mn}, \mathrm{Fe}, \mathrm{Co}, \mathrm{Ni}, \mathrm{Cu}$; see also Table 1). Lithium is a relatively rare element which was not detected before in any comet. An upper limit was reported for comet Ikeya-Seki (Table 1). It was observed, however, in the spectra of some meteoroids (e.g., in the Benešov bolide; Borovička \& Spurný 1995).

Since, due to sedimentation, metallic compounds are only expected to be present at very deep levels in Jupiter's atmosphere, there is little doubt that the atomic lines in the SL9 plumes are coming from the impactor itself. Translating the observed line intensities into elemental abundances requires the knowledge of the emission mechanism.

Fluorescence is the usual mechanism invoked to explain cometary atomic and molecular emissions, as well as the sodium emission lines observed in Io's torus. As far as the 

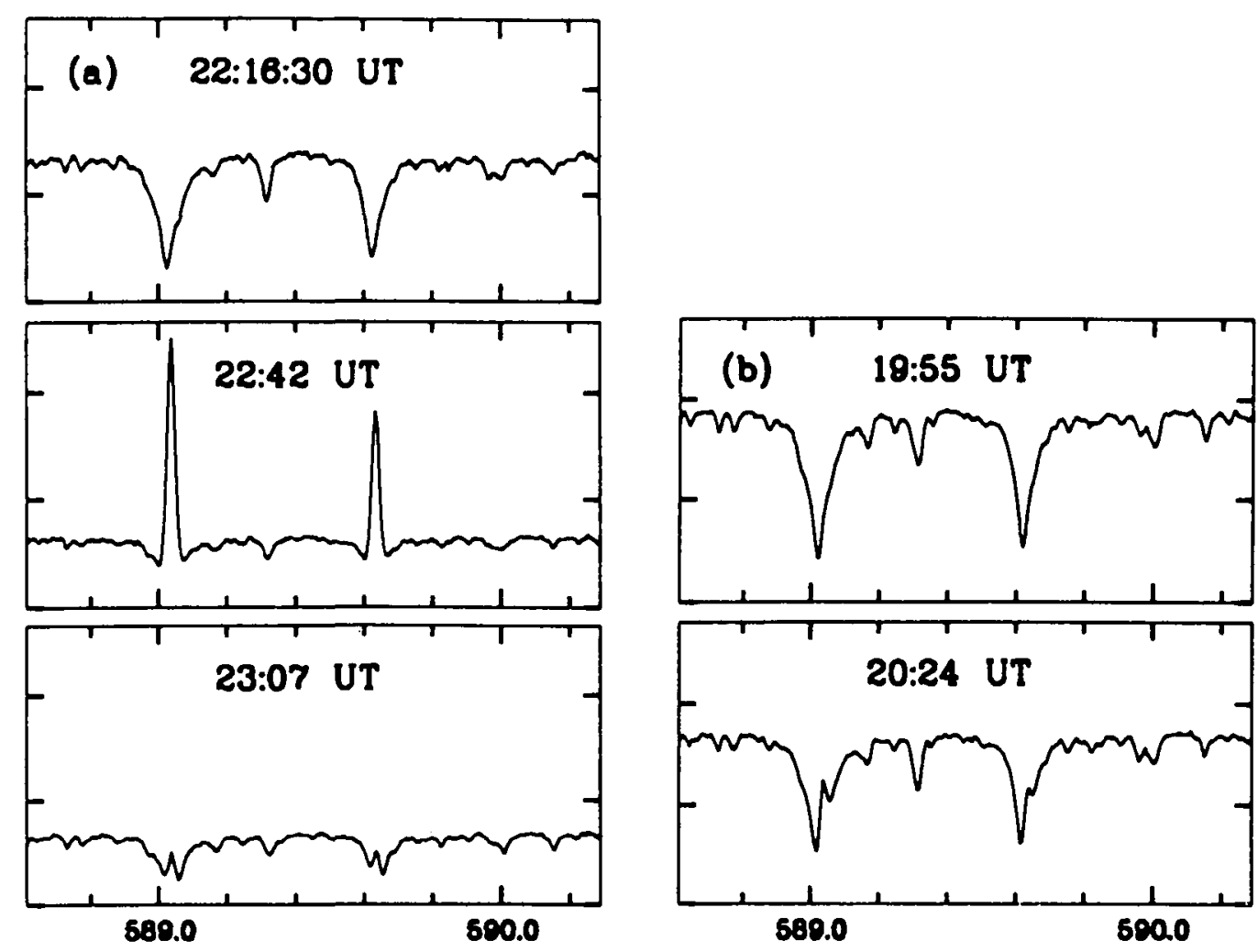

FIgURE 6. A time sequence showing the evolution of the $\mathrm{Na}$ emission line doublet during the $\mathrm{L}$ impact (left) and the $\mathrm{Q}$ impacts (right), observed at the 2-m telescope of the Pic-du-Midi observatory. The raw spectra are shown here, uncorrected for the reflected solar spectrum. The wavelength is given in nanometres. From Roos-Serote et al. (1995b).

lines are not saturated, the line intensities $I$ are proportional to the column densities $\langle N\rangle$ through the simple law $I=g\langle N\rangle$, where $g$ is the usual $g$-factor for resonant fluorescence. Saturated lines cannot exceed $I=\Phi \Delta \nu$, where $\Phi$ is the solar flux density at Jupiter and $\Delta \nu$ is the line width. This intensity was exceeded for the lines observed by the IUE (the $\mathrm{H}$ Lyman $\alpha, \mathrm{C}$ and possibly $\mathrm{Al}^{+}$lines, as reported by Ballester et al. 1995) as well as for most lines observed in the visible. For the HST observations, Noll et al. (1995) initially proposed emission due to resonant fluorescence. The observed line intensities, at least for the $\mathrm{Mg}$ and $\mathrm{Mg}^{+}$lines, are however large and could only be explained by saturated lines of width of $5 \mathrm{~km} \mathrm{~s}^{-1}$. This is significantly broader than the thermal line width (about $1.0 \mathrm{~km} \mathrm{~s}^{-1}$ at $1000 \mathrm{~K}$ ) or the width due to bulk motions within the impact sites (presumably $<1 \mathrm{~km} \mathrm{~s}^{-1}$ ). This suggests that the lines are broadened by heavy saturation (optical depths of the order of $10^{6}$ are necessary to broaden the lines by a factor of 4). Noll et al. derived column abundances and masses based upon the law $I=g\langle N\rangle$, valid for the optically thin case. The derived masses could thus be underestimated by many orders of magnitudes. They could still be consistent, however, with the elemental masses contained in a $10^{14}-10^{15} \mathrm{~g}$ fragment with cometary atomic abundances. It is unfortunate that in this heavily saturated situation, atomic abundances cannot be retrieved with any degree of accuracy. 


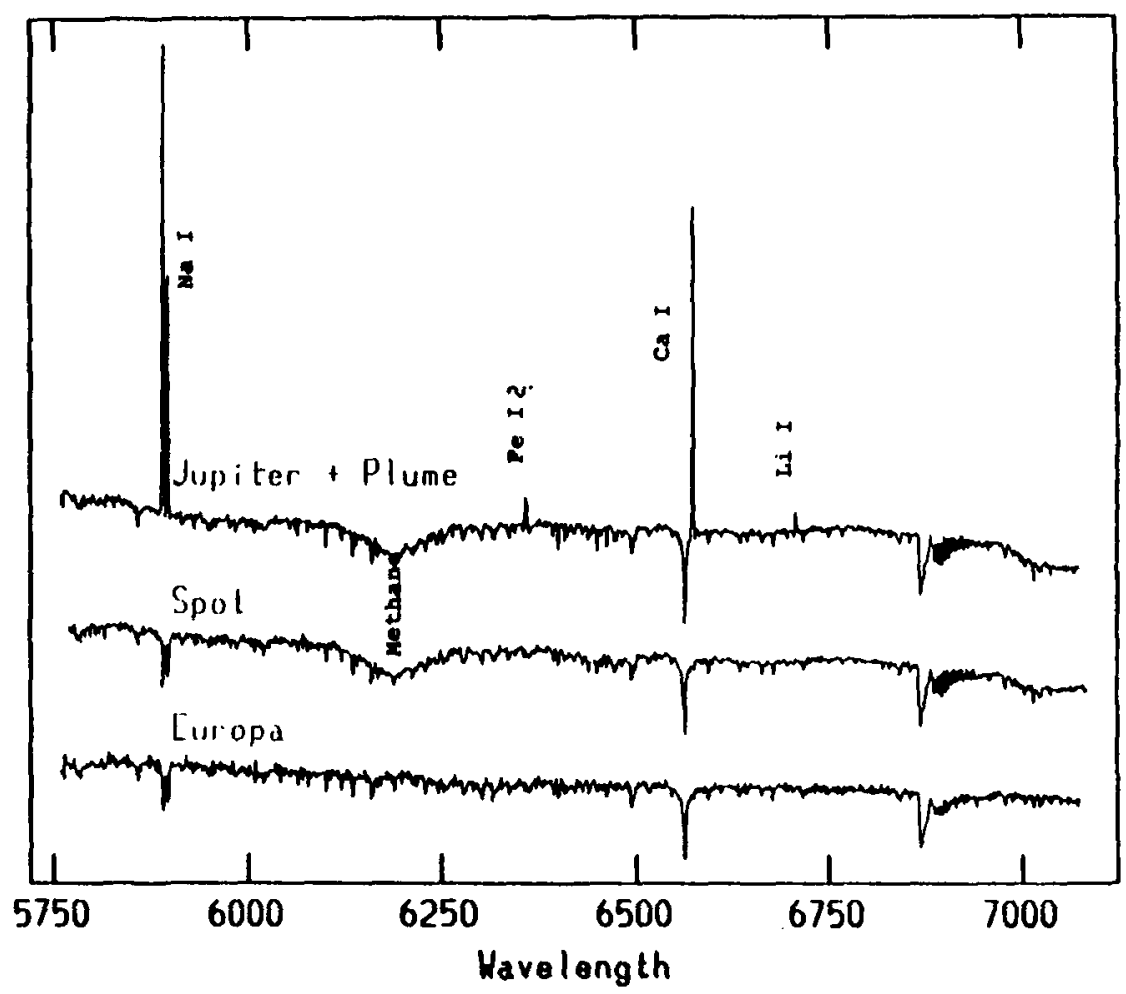

FIGURE 7. Spectrum in the 575-700 $\mathrm{nm}$ region of the plume of the L-impact observed with the 1.6-m telescope of the Laboratório Nacional de Astrofísica at Brazópolis. Emission lines of $\mathrm{Na}$, $\mathrm{Fe}, \mathrm{Ca}$ and $\mathrm{Li}$ are present. Below are shown, for comparison, the spectrum of the same region one hour later and that of Europa. The wavelength is given in angstrøms. From Costa et al. (1994).

There are several pieces of evidence suggesting that fluorescence from atoms in the ground state is not the mechanism (or not the only one) responsible for these line emissions. As noted by Carpenter et al. (1995), several of the lines observed in the UV are terminating at excited levels significantly above ground. In the visible, a strong intersystem $\mathrm{Ca}$ line at $657.3 \mathrm{~nm}$ is observed, whereas the fundamental Ca line at $422.8 \mathrm{~nm}$ is not detected in the La Palma spectra (Fig. 5).

Thermal excitation by collisions is a plausible mechanism for the transient emission of atomic lines. It can explain the spectrum of meteors in the Earth atmosphere, and it may be noted that the velocity of the splashback impacts on Jupiter was $\simeq 10 \mathrm{~km} \mathrm{~s}^{-1}$, of the same order as the entry velocities of meteors in the Earth atmosphere. Other possible mechanisms are electronic recombinations and prompt emissions following chemical reactions. The modelling of these time-dependent excitation phenomena requires the knowledge of the time evolution of the plume temperature, of the plume and impact site geometry and its evolution (to account for beam dilution), and of course of the line saturation. 


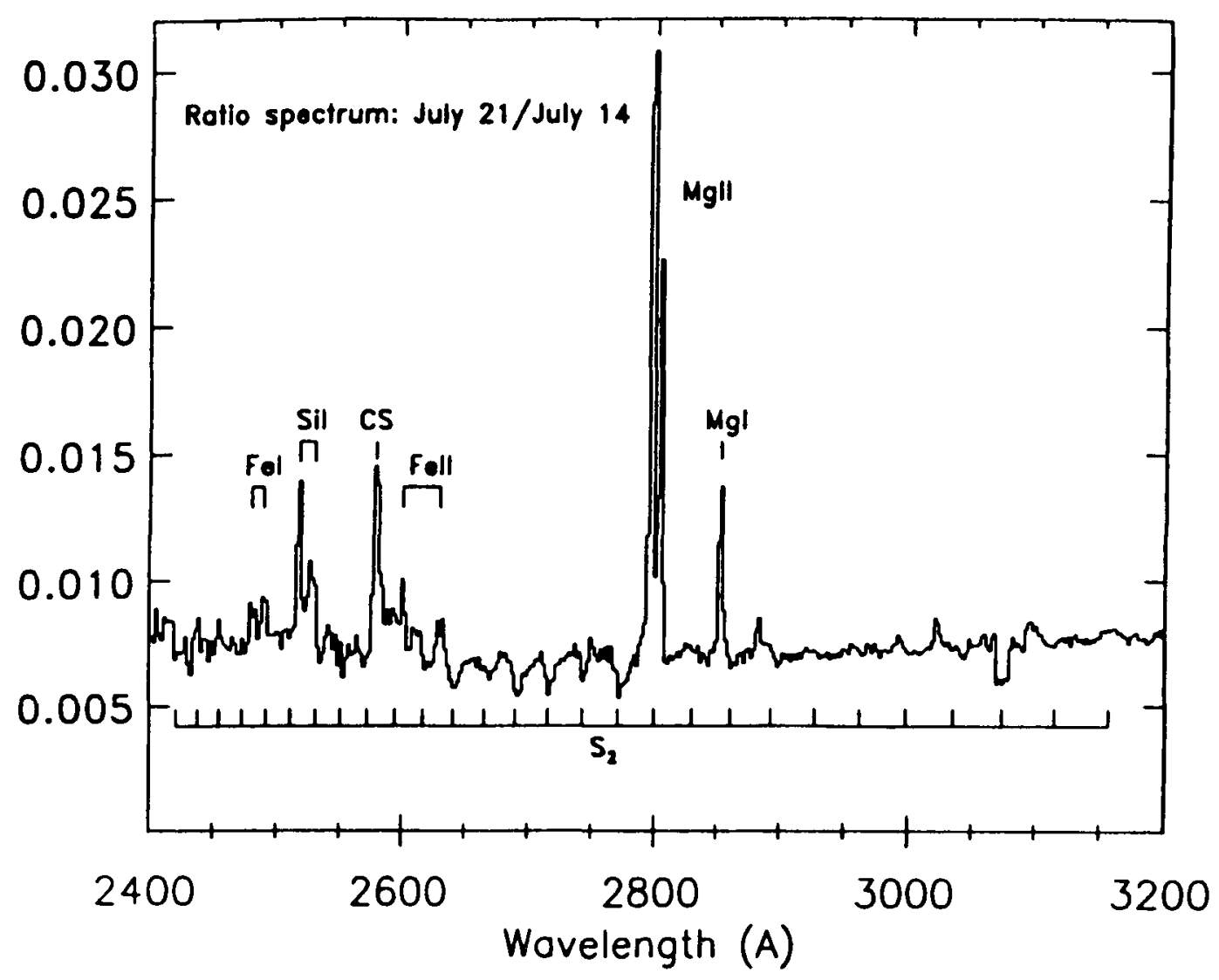

Figure 8. Atomic lines observed in the $240-320 \mathrm{~nm}$ region with the HST on the S impact site, $45 \mathrm{~min}$ after the impact. The spectrum has been ratioed by a pre-impact Jupiter spectrum. Weak unidentified emission lines are present around $300 \mathrm{~nm}$. From Noll et al. (1995).

\subsection{Clues from molecular lines}

Molecular lines and bands detected following the SL9 impacts on Jupiter are reported in Tables 3 and 4 . These observations are discussed in detail in the chapter by Lellouch (this volume). Some of these "detections" should be taken with caution, because they rely on a single instrument observation with a poor signal-to-noise ratio (like the radio observation of $\mathrm{H}_{2} \mathrm{O}$ ), or on a broad spectral feature which cannot provide an unambiguous identification (like $\mathrm{H}_{2} \mathrm{O}$ in the near-infrared or $\mathrm{H}_{2} \mathrm{~S}$ in the UV).

During the SL9 impact observing campaign, special attention was paid to the searches for water signatures, since it was believed that the presence or absence of this species in the impact sites would betray the nature of the impactor, comet nucleus or asteroid. Such signatures, which could not be found in the quick-look inspections, were only revealed after careful analysis of the data. Infrared spectroscopy revealed transient emissions of water lines just after the impacts (Table 3). The KAO observed emission lines of water at $7.67-7.72 \mu \mathrm{m}$ ( $\nu_{2}$ ro-vibrational lines) and at $22.6-23.9 \mu \mathrm{m}$ (rotational lines) just after the impacts of fragments $\mathrm{G}$ and $\mathrm{K}$ (Bjoraker et al. 1995) and at $6.6 \mu \mathrm{m}$ after impacts of $\mathrm{R}$ and $\mathrm{W}$ (Sprague et al. 1995). These lines correspond to high-energy rotational levels. They rapidly decreased in intensity and disappeared, which can be attributed to 
cooling of the gas rather than to the disappearance of water. Sprague et al. estimated the water masses corresponding to these observations to be 1.4 and $2.8 \times 10^{12} \mathrm{~g}$ for $\mathrm{G}$ and $\mathrm{K}$ (Bjoraker et al.), 6 and $10 \times 10^{10} \mathrm{~g}$ for $\mathrm{R}$ and $\mathrm{W}$ (Sprague et al.), equivalent to pure ice spheres of 140,180, 50 and $60 \mathrm{~m}$ in diameter, respectively. Observations of several impacts at the AAT revealed a rapidly varying infrared spectrum which could be fitted with transient emission of the $\nu_{3}$ and $\nu_{2}+\nu_{3}$ water bands (Crisp \& Meadows 1995). The observers of Galileo/NIMS also claim to have observed the $2.7 \mu \mathrm{m}$ water band in the splashbacks of $\mathrm{G}$ and $\mathrm{R}$, despite very poor spectral resolution (Carlson et al. 1995). A possible detection of the water line at $22 \mathrm{GHz}$ in the weeks following the impacts has also been reported (Montebugnoli et al. 1995).

The various observers of molecules detected at UV, infrared, and radio wavelengths, following the impacts have reported masses (or masses upper limits) that may strongly disagree (by orders of magnitudes!) from one technique to another. This should not be a surprise, since we are still in a preliminary state of analysis and:

- we are sometimes comparing different impacts;

- we are dealing with either transient effects observed in the plume or the rapidly cooling impact site (UV, IR), or medium- or long-term effects (radio);

- we have to take care of the different geometries (radio observations were affected by beam-dilution and encompassed several impact sites; UV observations may give lower limits due to limited sampling);

- line saturation is a problem which has to be considered (e.g., for fluorescence emission; see Section 4.2);

- the different observations were sounding different levels and the vertical distributions of the molecular species are poorly known; and

- assumptions must be made on the vertical temperature profiles (for the radio observations) and on the temperature evolution (for the UV and IR observation of the rapidly cooling impact sites).

Table 5 lists the masses of the various molecules observed in the major impact sites, adopted from the "baseline" values reported in the chapter by Lellouch (this volume); the reader is referred to this chapter for all details on their determination and their reliability. For the sake of discussion, Table 5 also lists the corresponding masses for several key elements contained in these molecules, as well as those contained in a comet or a CI chondrite of $10^{15} \mathrm{~g}$ mass, for the typical abundances of Table 1. The mass of $10^{15} \mathrm{~g}$ roughly corresponds to a kilometre-size fragment of density 1 , and is probably the upper bound of the plausible mass of the largest SL9 fragments.

Little can be learned from the mass of carbon, because most of this element was presumably provided by Jovian methane. In contrast, the mass of oxygen gives perhaps the most stringent constraint on the impactor: the upper atmosphere of Jupiter is devoid of oxygen compounds and it seems improbable that the impactors hit the hypothetical deep water clouds. Oxygen from CO alone amounts to $1.5 \times 10^{14} \mathrm{~g}$, corresponding to a fragment mass of at least $3 \times 10^{14} \mathrm{~g}$, if having either a cometary or a CI chondritic composition. To this, one should add the mass of oxygen contained in water: this latter is difficult to estimate since only "hot" water was detected in the plumes or the splashbacks. Cold water or condensed water ice (which might be an important component of the haze, unfortunately without specific spectral signature) eluded detection. Assuming that the mass of the initial nucleus was about ten times that of the big fragments, we obtain a lower limit of $3 \times 10^{15} \mathrm{~g}$ for the mass of the nucleus, corresponding to a diameter of $2 \mathrm{~km}$ (for a density of $0.5 \mathrm{~g} \mathrm{~cm}^{-3}$ ).

The sulphur mass is poorly known, mainly due to the still uncertain $\mathrm{S}_{2}$ mass and to that of OCS, a molecule only marginally detected in the radio. Given these uncertainties, 


\begin{tabular}{|c|c|c|c|c|c|}
\hline molecule & observation $^{\dagger}$ & $\begin{array}{l}\text { molecular } \\
\text { mass }{ }^{\dagger} \text { in a } \\
\text { SL9 impact }\end{array}$ & $\begin{array}{l}\text { elemental } \\
\text { mass in a } \\
\text { SL9 impact }\end{array}$ & $\begin{array}{l}\text { elemen } \\
\text { in a } 10^{15} \\
\text { comet }\end{array}$ & $\begin{array}{l}\text { al mass } \\
\text { fragment } \\
\text { CI-chondrite }\end{array}$ \\
\hline $\begin{array}{l}\mathrm{H}_{2} \mathrm{O} \\
\mathrm{CO}\end{array}$ & $\begin{array}{l}\text { IR, radio } \\
\text { IR, radio }\end{array}$ & $\begin{array}{l}>2 \times 10^{12} \\
2.5 \times 10^{14}\end{array}$ & $>1.5 \times 10^{14}[\mathrm{O}]$ & $5 \times 10^{14}[\mathrm{O}]$ & $5 \times 10^{14}[\mathrm{O}]$ \\
\hline $\begin{array}{l}\mathrm{NH}_{3} \\
\mathrm{HCN}\end{array}$ & $\begin{array}{l}\mathrm{UV}, \mathrm{IR} \\
\mathrm{IR} \text {, radio }\end{array}$ & $\begin{array}{l}1 \times 10^{13} \\
6 \times 10^{11}\end{array}$ & $1 \times 10^{13}[\mathrm{~N}]$ & $2 \times 10^{13}[\mathrm{~N}]$ & $3 \times 10^{11}[\mathrm{~N}]$ \\
\hline \multirow[t]{2}{*}{$\begin{array}{l}\mathrm{S}_{2} \\
\mathrm{CS}_{2} \\
\mathrm{CS} \\
\mathrm{H}_{2} \mathrm{~S} \\
\mathrm{OCS}\end{array}$} & $\begin{array}{l}\text { UV } \\
\text { UV } \\
\text { UV, radio } \\
\text { UV } \\
\text { radio }\end{array}$ & $\begin{array}{c}1.5 \times 10^{12} ? \\
1.5 \times 10^{11} \\
5 \times 10^{11} \\
? \\
3 \times 10^{12}\end{array}$ & $5 \times 10^{12}[\mathrm{~S}]$ & $4 \times 10^{13}[S]$ & $6 \times 10^{13}[\mathrm{~S}]$ \\
\hline & & & $1 \times 10^{14}[\mathrm{C}]$ & $2 \times 10^{14}[\mathrm{C}]$ & $3 \times 10^{13}[C]$ \\
\hline $\mathrm{PH}_{3}$ & IR & $?$ & $3 \times 10^{11}[\mathrm{P}]$ & $1 \times 10^{12}[\mathrm{P}]$ & \\
\hline silicates & IR & $6 \times 10^{12}$ & $8 \times 10^{13}[\mathrm{Si}]$ & $\begin{array}{l}1 \times 10^{14}[\mathrm{Si}] \\
4 \times 10^{13}[\mathrm{Mg}]\end{array}$ & $1 \times 10^{14}[\mathrm{Mg}]$ \\
\hline
\end{tabular}

$\dagger$ In bold are the observations from which the abundances are taken.

$\ddagger$ Some of these values are preliminary and subject to revision. See Tables 3 and 4 for references and Lellouch (this volume) for a discussion.

TABLE 5. Amounts (in g) of detected molecules and corresponding elemental masses following the impacts of SL9 on Jupiter, with a comparison with elemental masses expected from a comet nucleus or a carbonaceous chondrite.

the $[\mathrm{S}] /[\mathrm{O}]$ ratio is compatible with cometary or asteroidal abundances, and there is no need to invoke a contribution from the hypothetical $\mathrm{NH}_{4} \mathrm{SH}$ Jovian cloud (as it was by Noll et al. [1995], on the basis of a much higher preliminary estimation of the $\mathrm{S}_{2}$ mass).

Is it possible to discriminate between an impactor of cometary or asteroidal origin from these elemental abundances? The oxygen abundances are nearly the same in a cometary nucleus or in a primitive asteroid with composition supposed to be similar to that of carbonaceous chondrites; most of the oxygen is in water ice and dust silicates in the comet, in crystallization water and in silicates in the asteroid. Thus, the observed oxygen mass can inform us on the mass of the fragments, but not on their origin.

Light elements, however, such as $\mathrm{H}, \mathrm{C}, \mathrm{N}$, are significantly depleted in primitive asteroids (they could be still more depleted in more evolved asteroids). Nothing could be learned from $\mathrm{C}$ or $\mathrm{H}$, which could come from Jovian $\mathrm{H}_{2}$ and $\mathrm{CH}_{4}$. The nitrogen mass is important and corresponds to $[\mathrm{N}] /[\mathrm{O}] \simeq 0.07$; this is compatible with a cometary composition $([\mathrm{N}] /[\mathrm{O}] \simeq 0.04)$, but is much larger than a chondritic composition $([\mathrm{N}] /[\mathrm{O}]$ $\left.\simeq 6 \times 10^{-4}\right)$. However, most of the observed nitrogen is coming from ammonia: this argument would be ruined if this ammonia were coming from the $\mathrm{NH}_{3}$ cloud hit by the impactor.

It would also be interesting to know the $\mathrm{D} / \mathrm{H}$ ratio for the molecules observed in the impact sites. This could allow us to distinguish cometary material from processed 
Jovian material, since deuterium is believed to be heavily enriched in comets. D/H was measured to be $3 \times 10^{-4}$ for the water of $\mathrm{P} /$ Halley (Balsiger et al. 1995; Eberhardt et al. 1995) whereas it is $\simeq 2 \times 10^{-5}$ in Jupiter (Lécluse et al. 1995), similar to the protosolar value. Unfortunately, no information on isotopic ratios is available from spectroscopy of the SL9 impacts.

\subsection{Clues from aerosols}

The impacts were followed by the apparition of spots, or blemishes, that persisted for months for the strongest impacts. These spots were attributed to the formation of dark aerosols (see the chapters by Hammel and West).

Several authors attempted to derive the impactor's masses from the observations of the aerosols in the impact sites (Field et al. 1994; Knacke \& A'Hearn 1994; Tejfel et al. 1995; Vanysek 1995; R. A. West et al. 1995). The observations give directly the spot sizes (5000 to $15000 \mathrm{~km}$ diameter) and the filling factors (equal to the optical depth, 0.01 to 0.3 ) of the aerosol particles. With reasonable assumptions of the density $(0.5$ to $\left.3 \mathrm{~g} \mathrm{~cm}^{-3}\right)$ and the size $(0.5$ to $10 \mu \mathrm{m})$ of the particles, one can derive the total mass of the visible acrosols: $3 \times 10^{10}$ to $10^{15} \mathrm{~g}$ in a single impact site. To further obtain the masses of the impactors, one has then to estimate the fraction of the refractories which effectively recondense into aerosols, and the fraction of the impactor which is composed of refractories. Due to the large uncertainties on all parameters, we must admit that these masses are not seriously constrained.

The nature of the aerosols is still the subject of debates. Multi-wavelength observations permitted measurement of the imaginary part of the refractive index of the aerosol material, and thus a constraint on its nature (R. A. West et al. 1995). The dark brown colour suggests graphite, dirty silicates, organics, sulphur and nitrogen compounds.

A key observation was the identification of the silicate emission feature at $10 \mu \mathrm{m}$ in a spectrum obtained immediately after impact $\mathrm{R}$ at Palomar observatory (Nicholson et al. 1995). It corresponded to a mass of silicates of $6 \times 10^{12} \mathrm{~g}$, assuming grains of radius $\leq 1 \mu \mathrm{m}$ and density $3.3 \mathrm{~g} \mathrm{~cm}^{-3}$.

Recondensed refractories are thus highly plausible candidates. Models of recondensation of silicates have been achieved by Friedson (1995) and Hasegawa et al. (1995). Poly-HCN has also been proposed because its imaginary refractive index closely reproduces that observed (R. A. West, this volume, R. A. West et al. 1995); it can be noted that $\mathrm{HCN}$ is an identified component of both cometary nuclei and the impact sites (Marten et al. 1995). A chemical model by Lyons (1995), however, showed that poly-HCN is unlikely to form in the post-impact reactions. Wilson \& Sagan (1995) remarked that the observed imaginary refractive index is also close to that of the organic residue of the Murchison carbonaceous chondrite, and they proposed that the aerosols could come from chemically preserved debris of the impactor.

It is probable that the aerosol is of a complex composition and that its grains are heterogeneous due to the variety of the input material. It should also be stressed that the aerosols may consist, in a large part, of ices of water, ammonia and carbon dioxide, which escaped detection because of their small absorption coefficients.

\section{Conclusion}

Retrieving the chemical composition of SL9 from the spectroscopic observations of the SL9 event is a difficult problem. A careful modelling of the physical conditions of the impacts (geometry, temperature, time evolution) is required, as well as of the chemical processes. There is no doubt that these challenging issues could only be solved by the 
close collaboration of all observers and modellers, in order to put all the pieces of the puzzle together.

At the present stage of the interpretation of the observations, SL9 appears to be a very brittle body for which we have only soft constraints on the composition. These constraints agree with our current knowledge of the cometary and asteroidal compositions. Trying to classify SL9 as a comet or an asteroid is perhaps a meaningless point: we now know that these two kinds of small bodies are closely related and that they may evolve from one class to another.

Therefore, it seems appropriate to conclude by the following quotations:

There is no compelling need to invoke a special class of object for SL9. The classification of SL9 as a presumably normal Jupiter-family short-period comet is not contradicted by any observation so far. (R. M. West 1995.)

Most of the objects classified as asteroids at and beyond Jupiter's orbit are likely to conceal buried volatiles, and thus are more usefully considered as comets. (Jewitt 1995b.)

I would like to thank all the observers of comet SL9 and of its impacts on Jupiter who communicated their results prior to publication, and the editors for their comments and their help in improving this paper.

\section{REFERENCES}

A'Hearn, M. F., Millis, R. L., Schleicher, D. G., Osip, D. J. \& Birch, P. V. 1995 The ensemble properties of comets: results from narrowband photometry of 85 comets, 1976-1992. Icarus, 118, 223-270.

A'Hearn, M. F., Schleicher, D. G., Feldman, P. D., Millis, R. L. \& Thompson, D. T. 1984 Comet Bowell 1980b. Astron. J. 89, 579-591.

ANDERs, R. \& Grevesse, E. 1989 Abundances of the elements: meteoritic and solar. Geochim. Cosmochim. Acta 53, 197-214.

ARPIGNY, C. 1979 Relative abundances of the heavy elements in comet Ikeya-Seki (1965 VIII). Mem. Soc. Roy. Liège 22, 189-197.

ARPIGNY, C. 1994 Physical chemistry of comets: models, uncertainties, data needs. In Molecules and Grains in Space, (ed. I. Nenner), AIP Conference Proceedings 312, pp. 205-238.

Arpigny, C., Dossin, F., WoszczYK, A. et al. 1995 Atlas of cometary spectra. Kluwer (in press).

Atreya, S. K., Edgington, S. G., Trafton, L. M. et al. 1995 Abundance of ammonia and carbon disulfide in the Jovian stratosphere following the impact of comet ShoemakerLevy 9. Geophys Res. Let. 22, 1625-1628.

Ballester, G. E., Harris, W. M., Gladstone, G. R. et al. 1995 Far-UV emissions from the SL9 impacts with Jupiter. Geophys. Res. Let. 22, 2425-2429.

Balsiger, H., Altwegg, K. \& Geiss, J. $1995 \mathrm{D} / \mathrm{H}$ and ${ }^{18} \mathrm{O} /{ }^{16} \mathrm{O}$-ratio in the hydronium ion and in neutral water from in situ ion measurements in comet Halley. J. Geophys. R. 100, $5827-5834$.

Belton, M. J. S., Chapman, C. R. et al. 1995 Bulk density of asteroid 243 Ida from the orbit of its satellite Dactyl. Nature 374, 785-788.

BÉzard, B., Griffith, C. A., Kelly, D. et al. 1995 Mid-IR high-resolution spectroscopy of the SL9 impact sites: temperature and $\mathrm{HCN}$ retrievals. IAU Coll. No. 156 (poster).

Buoraker, G. L., Stolovy, S. R., Herter, T. L., Gull, G. E. \& Pirger, B. E. 1995 Detection of water after the collision of fragments $G$ and $K$ of comet Shoemaker-Levy 9 with Jupiter. Icarus (submitted).

Bockelée-Morvan, D., Colom, P., Despois, D. et al. 1995 Observations of the ShoemakerLevy 9 impacts on Jupiter at the Swedish-ESO submillimetre telescope. The Messenger 79, 29-31. 
BorovičKA J. \& SPURNÝ P. 1995 Radiation study of two very bright terrestrial bolides. Icarus (submitted).

Borunov, S., Drossart, P., Encrenaz, T. \& Dorofeeva, V. 1995 High temperature chemistry in the fireballs formed by the impacts of comet P/Shoemaker-Levy 9 in Jupiter. Icarus (submitted).

Carlson, R. W., Weissman, P. R., Segura, M. et al. 1995 Galileo infrared observations of the Shoemaker-Levy $9 \mathrm{G}$ impact fireball: a preliminary report. Geophys Res. Let. 22, $1557-1560$.

Carpenter, K. G., McGrath, M. A., Yelle, R. V., Noll, K. S. \& Weaver, H. A. 1995 Formation of atomic emission lines in the atmosphere of Jupiter after the comet ShoemakerLevy $9 \mathrm{~S}$ impact. Bull. Amer. Astron. Soc., 27, 64.

Catalano, S., Rodono, M., Ventura, R. \& Cacciani, A. 1995 Observations of the SL9 impacts with a sodium magneto-optical filter. European SL-9/Jupiter Workshop, (eds. R. M. West and H. Boehnhardt), ESO Conference and Workshop Proceedings No. 52, pp. 209-214.

Chernova, G. P., Jockers, K. \& Kiselev, N. N. 1995 Imaging photometry and color of comet Shoemaker-Levy 9. Icarus (submitted).

Cochran, A. L., Whipple, A. L., MacQueEn, P. J. et al. 1994 Preimpact characterization of comet $\mathrm{P} /$ Shoemaker-Levy 9 . Icarus 112, 528-532.

Colas, F., Jorda, L., LeCAcheuX, J. et al. 1995 Pre-impact observations of ShoemakerLevy 9 at Pic du Midi and Observatoire de Haute Provence. European SL-9/Jupiter Workshop, (eds. R. M. West and H. Boehnhardt), ESO Conference and Workshop Proceedings No. 52, pp. 23-28.

Conrath, B. J., Gierasch, P. J., Hayward, T. et. al. 1995 Palomar mid-infrared observations of comet Shoemaker-Levy 9 impact sites. IAU Coll. No. 156 (poster).

Copeland, R. \& LohSE, J. G. 1882 Spectroscopic observations of comets III and IV, 1881, comet I, 1882, and the Great Comet of 1882. Copernicus 2, 225-244.

Costa, R. D. D., de Freitas Pacheco, J. A., Singh, P. D., De Almeda, A. A. \& Codina, S. J. 1994 Detection of lithium in the plume of the L-fragment impact of comet ShoemakerLevy 9 with Jupiter. Preprint.

CRISP, D. \& MEADOWs, V. 1995 Near-infrared imaging spectroscopy of the impacts of SL9 fragments C, D, G, K, N, R, V, and W with Jupiter. IAU Coll. No. 156 (poster).

Crovisier, J. 1994. Molecular abundances in comets. In Asteroids, Comets, Meteors 1993, (eds. A. Milani et al.). pp. 313-326. Kluwer.

Crovisier, J., Biver, N., Bockelée-Morvan, D. et al. 1995 Carbon monoxide outgassing from comet $\mathrm{P} / \mathrm{Sch}$ assmann-Wachmann 1. Icarus 115, 213-216.

Eberhardt, P., Regber, M., Krankowsky, D. \& Hodges, R. R. 1995 The D/H and ${ }^{18} \mathrm{O} /{ }^{16} \mathrm{O}$ ratios in water from comet $\mathrm{P} /$ Halley. Astron. Astrophys.

Feldman, P. D., Weaver, H. A., Boice, D. C. \& Stern, S. A. 1995 HST observations of $\mathrm{Mg}^{+}$in outburst from comet D/Shoemaker-Levy 9. Icarus (in press).

Festou, M. C., Rickman, H. \& West, R. M. 1993 Comets. Astron. Astrophys. Reviews 3, $363-447$ and $5,37-163$.

Field, G. B., Tozzi, G. P. \& Stanga, R. M. 1994. Dust as the cause of spots on Jupiter. Astron. Astrophys. 294, L53-L55.

Fitzsimmons, A., Litrle, J. E., Andrews, P. J. et al. 1995a Optical imaging and spec-troscopy of the impact plumes on Jupiter. European $S L-9 / J u p i t e r$ Workshop, (eds. R. M. West and H. Boehnhardt), ESO Conference and Workshop Proceedings No. 52, pp. 197201.

FRIEDSON, A. J. 1995 Refractory grain formation in Shoemaker-Levy fireballs. Icarus (submitted).

Gladstone, G. R., Hall, D. T. \& Waite, JR., J. H. 1995 EUVE observations of Jupiter during the impact of comet Shoemaker-Levy 9. Science 268, 1595-1597. 
GRIFFITH, C. A., BÉZARD, B., KELLY, D. et al. 1995. Mid-IR spectroscopy and $\mathrm{NH}_{3}$ and $\mathrm{HCN}$ images of $\mathrm{K}$ impact site. IAU Coll. No. 156 (poster).

Hammel, H. B., BeEbe, R. F., Ingersoll, A. P. et al. 1995 HST imaging of atmospheric phenomena created by the impact of comet Shoemaker-Levy 9. Science 267, 1288-1296.

Hasegawa, H., Takeuchi, S., Yamashita, T. \& Watanabe, J. 1995 Reflectivities of the cometary impact sites. IAU Coll. No. 156 (poster).

Hoffmann, M. 1989. Asteroid mass determination: present situation and perspectives. In $A s-$ teroids II, (eds. R. P. Binzel et al.), pp. 228-239. University of Arizona Press.

Huebner, W. F., Keller, H. U., Jewitt, D., Klinger, J. \& West, R. (eds.) 1993 Workshop on the Activity of Distant Comets. SWRI.

Jessberger, E. K. \& Kissel, J. 1991 Chemical properties of cometary dust and a note on carbon isotope. In Comets in the Post-Halley Era, (eds. R. L. Newburn et al.), pp. 10751092. Kluwer.

JEWITT, D. 1995a Pre-impact observations of SL-9. European SL-9/Jupiter Workshop, (eds. R. M. West and H. Boehnhardt), ESO Conference and Workshop Proceedings No. 52, pp. 1-4.

JEWITT, D. 1995b From comets to asteroids: when hairy stars go bald. In Small Bodies in the Solar System and their Interactions with the Planets, (eds. H. Rickman and M. Dahlgren). Kluwer (in press).

KNACKe, R. F.\& A'Hearn, M. F. 1994 An estimate of the comet Shoemaker-Levy 9 fragment sizes from the debris fields. Earth Moon Planets 66, 11-12.

LÉcluse, C., Robert, F., Gautier, D. \& Guiraud, M. 1995 Laboratory determinations of deuterium exchange kinetics: application to the determination of the $\mathrm{D} / \mathrm{H}$ ratio in giant planets, Icarus (in press).

Lellouch, E., Paubert, G., Moreno, R. et al. 1995 Chemical and thermal response of Jupiter's atmosphere following the impact of comet Shoemaker-Levy 9 . Nature 373, 592595.

LyONS, J. R. 1995. Thermochemical and kinetic modeling of the SL9 impact debris. IAU Coll. No. 156 (poster).

Marten, A., Gautier, D., Owen, T. et al. 1995 The collision of the comet Shoemaker-Levy 9 with Jupiter: detection and evolution of HCN in the stratosphere of the planet. Geophys. Res. Lett. 22, 1589-1592.

Matthews, H. E., Jewitt, D. \& Senay, M. C. 1995 IAU Circ. No. 6234.

MEech, K. J. \& WeAver, H. A. 1995 Unusual comets(?) as observed from the Hubble Space Telescope. In Small Bodies in the Solar System and their Interactions with the Planets, (eds. H. Rickman and M. Dahlgren). Kluwer (in press).

Montebugnoli, S., Bortolotti, C., Cattani, A. et al. 1995 Detection of the 22-GHz line of water during and after the SL-9/Jupiter impact. European SL-9/Jupiter Workshop, (eds. R. M. West and H. Boehnhardt), ESO Conference and Workshop Proceedings No. 52, pp. 261-266.

Nicholson, P. D., Gierasch, P. J., Hayward, T. L. et al. 1995 Palomar observations of the $\mathrm{R}$ impact of comet Shoemaker-Levy 9: II. Spectra. Geophys. Res. Lett. 22, 1617-1620.

Noll, K. S., McGrath, M. A., Trafton, L. M. et al. 1995.. HST spectroscopic observations of Jupiter after the collision of comet P/Shoemaker-Levy 9. Science 267, 1307-1313.

Orton, G., A'Hearn, M., Baines, D. et al. 1995 Collision of comet Shoemaker-Levy 9 with Jupiter observed by the NASA Infrared Telescope Facility. Science 267, 1277-1282.

Prangé, R., Emerich, C., Talaver.a, A. et al. 1994 IAU Circ. No. 6041.

Preston, G. W. 1967 The spectrum of comet Ikeya-Seki (1965f). Astron. J. 147, 718-742.

RAUER, H. \& OSTERLOH, M. 1995. A search for differences in colour of the fragments of comet Shoemaker-Levy 9. Astron. Astrophys. 295, L31-L34.

Rauer, H., Despois, D., Moreno, R. et al. 1995 IAU Circ. No. 6236. 
Rickman, H. 1994 Cometary nuclei. In Asteroids, Comets, Meteors 1993, (eds. A. Milani et al.), pp. 297-312. Kluwer.

Roos-Serote, M., Barucci, A., Crovisier, J. et al. 1995a Metallic emission lines during the impacts L and $\mathrm{Q}_{1}$ of SL-9 on Jupiter. Geophys. Res. Let. 22, 1621-1624.

Roos-Serote, M., Barucci, A., Crovisier, J. et al. 1995b Pic-du-Midi observations of atomic lines following impacts L and $Q_{1}$ of comet SL-9 with Jupiter. European SL-9/Jupiter Workshop, (eds. R. M. West and H. Boehnhardt), ESO Conference and Workshop Proceedings No. 52, pp. 203-208.

Scotti, J. V., Gehrels, T. \& Metcalfe, T. S. 1994 Spacewatch observations of the surface brightness of comet $\mathrm{P} /$ Shoemaker-Levy 9 . Preprint.

Sekanina, Z. 1995a The splitting of the nucleus of comet Shoemaker-Levy 9. European SL9/Jupiter Workshop, (eds. R. M. West and H. Boehnhardt), ESO Conference and Workshop Proceedings No. 52, pp. 43-55.

Sekanina Z. 1995b Nuclei of SL-9 on images taken with the Hubble Space Telescope. European SL-9/Jupiter Workshop, (eds. R. M. West and H. Boehnhardt), ESO Conference and Workshop Proceedings No. 52, pp. 29-35.

Sekanina, Z., Chodas, P. W. \& Yeomans, D. K. 1994 Tidal disruption and the appearance of periodic comet Shoemaker-Levy 9. Astron. Astrophys. 289, 607-636.

SENAY, M. C. \& JEWITT, D. 1994 Coma formation driven by carbon monoxide release from comet Schwassmann-Wachmann 1. Nature 371, 229-231.

Sprague, A. L., BJoraker, G. L., Hunten, D. M. et al. 1995 Observations of $\mathrm{H}_{2} \mathrm{O}$ following the R and W impacts of comet SL-9 into Jupiter's atmosphere. Science (submitted).

Stüwe, J. A., Schulz, R. \& A'Hearn, M. F. 1995 NTT observations of SL-9: imaging and spectroscopy. European SL-9/Jupiter Workshop, (eds. R. M. West and H. Boehnhardt), ESO Conference and Workshop Proceedings No. 52, pp. 17-20.

TANCREDI, G. \& Lindgren, M. 1994 Searching for comets encountering Jupiter: first campaign. Icarus 107, 311-321.

Tejfel, V. G., Kharitonova, G. A., Sinyaeva, N. V. et al. 1995 The reflectivity of the aerosol material in the G, L and E impact-regions. IAU Coll. No. 156 (poster).

Trilling, D. E., Keller, H. U., Rauer, H., Schulz, R. \& Thomas, N. 1995 Observations of $\mathrm{P} /$ Shoemaker-Levy 9 in Johnson B, V and R filters from Calar Alto Observatory on 2/3 June, 1994. European SL-9/Jupiter Workshop, (eds. R. M. West and H. Boehnhardt), ESO Conference and Workshop Proceedings No. 52, pp. 37-41.

VANYSEK, V. 1995 A note on the fragments size of SL-9 and debris field. European SL-9/Jupiter Workshop, (eds. R. M. West and H. Boehnhardt), ESO Conference and Workshop Proceedings No. 52, pp. 297-298.

Weaver, H. A., A'Hearn, M. F., Arpigny, C. et al. 1995 The Hubble Space Telescope (HST) observing campaign on comet P/Shoemaker-Levy 9. Science 267, 1282-1288.

Weaver, H. A., Feldman, P. D., A'Hearn, M. F. et al. 1994 Hubble Space Telescope observations of comet P/Shoemaker-Levy 9 (1993e), Science 263, 787-791.

West, R. A., KarkoshKa, E., Friedson, A. J. et al. 1995 Impact debris particles in Jupiter's atmosphere. Science 267, 1296-1301.

WEST, R. M. 1995 Nature and Structure of the impacting objects. European SL-9/Jupiter Workshop, (eds. R. M. West and H. Boehnhardt), ESO Conference and Workshop Proceedings No. 52 , pp. $407-410$.

WEST, R. M., HooK, R. \& HaInaut, O. 1995. A morphological study of SL-9 CCD images obtained at La Silla (July 1-15, 1994). European SL-9/Jupiter Workshop, (eds. R. M. West and H. Boehnhardt), ESO Conference and Workshop Proceedings No. 52, pp. 5-10.

WILson, P. D. \& SAGAN, C. 1995 Chemistry of the Shoemaker-Levy 9 jovian impact blemishes: indigenous cometary vs. shock-synthesized organic matter. IAU Coll. No. 156 (poster).

Wink, J., Lucas, R., Guilloteau, S. \& Dutrey, A. 1994 Interferometric observations of HCN J=1-0 during the SL-9 crash on Jupiter. IR.AM Newsletter 18, 5. 
Wyckoff, S., Wagner, M., Wehinger, P., Schleicher, D. \& Festou, M. 1984 Onset of sublimation in comet P/Halley. Nature 316, 241-242.

XIE, X., Mumma, M. J. \& Olson, K. M. 1995 Physical properties of swarmed SL9 fragments at impact. IAU Coll. No. 156 (poster).

Yelle, R. V. \& MCGRATh, M. A. 1995 Ultraviolet spectroscopy of the SL9 impact sites, I: the $175-230 \mathrm{~nm}$ region. Icarus (submitted).

ZAhNLE, K. \& MAC Low, M.-M. 1995 A simple model for the light curve generated by a Shoemaker-Levy 9 impact. J. Geophys. Res. 100, 16885-16894. 